LMU-TPW 00-13

UWThPh-2000-19

LPTE Orsay 00/54

\title{
Field Theory on the $q$-deformed Fuzzy Sphere I
}

\author{
H. Grosse ${ }^{a \text { \#, J. Madore }}{ }^{b}$ and H. Steinackerç \\ ${ }^{a}$ Institut for Theoretical Physics, University of Vienna, \\ Boltzmanngasse 5, A-1090 Vienna, Austria \\ ${ }^{b}$ Max-Planck-Institut für Physik \\ Föhringer Ring 6, D-80805 München \\ and \\ Laboratoire de Physique Théorique et Hautes Energies \\ Université de Paris-Sud, Bâtiment 211, F-91405 Orsay \\ ${ }^{c}$ Sektion Physik der Ludwig-Maximilians-Universität München \\ Theresienstr. 37, D-80333 München
}

\begin{abstract}
We study the $q$-deformed fuzzy sphere, which is related to $D$-branes on $S U(2)$ WZW models, for both real $q$ and $q$ a root of unity. We construct for both cases a differential calculus which is compatible with the star structure, study the integral, and find a canonical frame of one-forms. We then consider actions for scalar field theory, as well as for Yang-Mills and Chern-Simons-type gauge theories. The zero curvature condition is solved.
\end{abstract}

Keywords: fuzzy sphere, quantum group, gauge theory

PACS classification 11.10.Kk, 03.65.Fd

MSC 81R50, 81T13, 58B30

\footnotetext{
${ }^{1}$ grosse@doppler.thp.univie.ac.at

2 John.Madore@th.u-psud.fr

${ }^{3}$ Harold.Steinacker@physik.uni-muenchen.de
} 


\section{Introduction}

There has been considerable work aimed at formulating models of quantum field theory on non-commutative spaces. The motivation is to obtain new insights into the UV-divergences and the problem of renormalization. On some simple noncommutative spaces, it is now possible to formulate quantum field theories. In some case, the UV divergences are completely regularized [16, 25], while in others they persist [13, 27]. Moreover, it was realized that such noncommutative spaces are in fact induced by certain sectors of string theory, particularly open strings ending on $D$-branes with a background $B$ field [7]. This is both a valuable source of physical insights, as well as a vindication of a more "puristic" approach of studying such spaces per se. In particular, spaces with quantum group symmetries have also been studied from a more formal approach. While quantum groups appear naturally in the context of 2-dimensional conformal field theories [3], a formulation of a quantum field theory based on such spaces has proved to be difficult.

Recently, Alekseev, Recknagel and Schomerus [1] have found that spherical $D$-branes in the $S U(2)$ WZW model are seen by open strings ending on them (in an appropriate background) as certain quasi-associative algebras, which are closely related to $q$-deformations of fuzzy spheres. Here $q$ is related to the level $k$ of the WZW model by the formula

$$
q=\exp \left(\frac{i \pi}{k+2}\right)
$$

We shall take this as sufficient motivation to study in detail the $q$-deformed fuzzy spheres, and to formulate field theories on them.

The algebra found in [四] is (weakly) non-associative, and covariant under $S U(2)$. Using a so-called Drinfeld twist, it can be transformed into an associative algebra which we call $\mathcal{S}_{q, N}^{2}$. It is covariant under the "quantum group" $U_{q}(s u(2))$, which is the quantized universal enveloping algebra of Drinfeld and Jimbo [9, 19]. Here $N$ is an integer related to a particular boundary condition on the $D$-brane in $S U(2)$ WZW model.

After reviewing the undeformed fuzzy sphere, we define $\mathcal{S}_{q, N}^{2}$ in Section 2 for both $q \in \mathbb{R}$ and $|q|=1$. As an algebra, it is simply a finite-dimensional matrix algebra, equipped with additional structure such as an action of $U_{q}(s u(2))$, a covariant differential calculus, a star structure, and an integral. For $q \in \mathbb{R}$, this is precisely the "discrete" series of Podleś spheres [29]. The case $|q|=1$, which is most relevant to string theory, has apparently not been studied in detail in the literature. In Section 3, we develop the non-commutative differential geometry on $\mathcal{S}_{q, N}^{2}$, using an approach which is suitable for both $q \in \mathbb{R}$ and $|q|=1$. The differential calculus turns out to be rather elaborate, but quite satisfactory. We are able to show, in particular, that in both cases there exists a 3-dimensional exterior differential 
calculus with real structure and a Hodge star, and we develop a frame formalism [12, 11, 26]. This allows us to write Lagrangians for field theories on $\mathcal{S}_{q, N}^{2}$. In particular, the fact that the tangential space is 3-dimensional unlike in the classical case turns out to be very interesting physically, and is related to recent results [2] on Chern-Simons actions on the $D$-branes.

Using these tools, we study in Section 4 actions for scalar fields and abelian gauge fields on $\mathcal{S}_{q, N}^{2}$. The latter case is particularly interesting, since it turns out that certain actions for gauge theories arise in a very natural way in terms of polynomials of one-forms. In particular, the kinetic terms arise automatically due to the noncommutativity of the space. Moreover, because the calculus is 3-dimensional, the gauge field consists of a usual (abelian) gauge field plus a (pseudo) scalar in the classical limit. This is similar to a Kaluza-Klein reduction. One naturally obtains analogs of Yang-Mills and Chern-Simons actions, again because the calculus is 3 -dimensional. In a certain limit where $q=1$, such actions were shown to arise from open strings ending on $D$-branes in the $S U(2)$ WZW model [2]. The gauge theory actions for $q \neq 1$ suggest a new version of gauge invariance, where the gauge "group" is a quotient $U_{q}(s u(2)) / I$, which can be identified with the space of functions on the deformed fuzzy sphere. This is discussed in Section 4.2.

Finally in Section 5, we give the precise relation of $\mathcal{S}_{q, N}^{2}$ to the quasi-associative algebra of functions on $D$-branes found in [1], using a Drinfeld-twist.

In this paper, we shall only consider the first-quantized situation; the second quantization is postponed to a forthcoming paper [18]. The latter turns out to be necessary for implementing the symmetry $U_{q}(s u(2))$ on the space of fields in a fully satisfactory way.

\section{The $q$-deformed fuzzy sphere}

\subsection{Review of the undeformed case}

We briefly recall the definition of the "standard" fuzzy sphere [25, 16]. Much information about the standard unit sphere $S^{2}$ in $\mathbf{R}^{\mathbf{3}}$ is encoded in the infinite dimensional algebra of polynomials generated by $\tilde{x}=\left(\tilde{x}_{1}, \tilde{x}_{2}, \tilde{x}_{3}\right) \in \mathbf{R}^{\mathbf{3}}$ with the defining relations

$$
\left[\tilde{x}_{i}, \tilde{x}_{j}\right]=0, \quad \sum_{i=1}^{3} \tilde{x}_{i}^{2}=r^{2}
$$


The algebra of functions on the fuzzy sphere is defined as the finite algebra $\mathcal{S}_{N}^{2}$ generated by $\hat{x}=\left(\hat{x}_{1}, \hat{x}_{2}, \hat{x}_{3}\right)$, with relations

$$
\left[\hat{x}_{i}, \hat{x}_{j}\right]=i \lambda_{N} \varepsilon_{i j k} \hat{x}_{k}, \quad \sum_{i=1}^{3} \hat{x}_{i}^{2}=r^{2} .
$$

The real parameter $\lambda_{N}>0$ characterizes the non-commutativity.

These relations are realized in a suitable finite-dimensional irreducible unitary representations of the $S U(2)$ group. This is most conveniently done using the Wigner-Jordan realization of the generators $\hat{x}_{i}, i=1,2,3$, in terms of two pairs of annihilation and creation operators $A_{\alpha}, A^{+\alpha}, \alpha= \pm \frac{1}{2}$, which satisfy

$$
\left[A_{\alpha}, A_{\beta}\right]=\left[A^{+^{\alpha}}, A^{+\beta}\right]=0,\left[A_{\alpha}, A^{+\beta}\right]=\delta_{\alpha}^{\beta},
$$

and act on the Fock space $\mathcal{F}$ spanned by the vectors

$$
\left|n_{1}, n_{2}\right\rangle=\frac{1}{\sqrt{n_{1} ! n_{2} !}}\left(A^{+\frac{1}{2}}\right)^{n_{1}}\left(A^{+-\frac{1}{2}}\right)^{n_{2}}|0\rangle
$$

Here $|0\rangle$ is the vacuum defined by $A_{i}|0\rangle=0$. The operators $\hat{x}_{i}$ take the form

$$
\hat{x}_{i}=\frac{\lambda_{N}}{\sqrt{2}} A^{+\alpha^{\prime}} \varepsilon_{\alpha^{\prime} \alpha} \sigma_{i}^{\alpha \beta} A_{\beta} .
$$

Here $\varepsilon_{\alpha \alpha^{\prime}}$ is the antisymmetric tensor (spinor metric), and $\sigma_{i}^{\alpha \beta}$ are the Clebsch-Gordan coefficients, that is rescaled Pauli-matrices. The number operator is given by $\hat{N}=\sum_{\alpha} A^{+\alpha} A_{\alpha}$. When restricted to the $(N+1)$-dimensional subspace

$$
\mathcal{F}_{N}=\left\{\sum A^{+\alpha_{1}} \ldots A^{+\alpha_{N}}|0\rangle(N \text { creation operators })\right\}
$$

it yields for any given $N=0,1,2, \ldots$ the irreducible unitary representation in which the parameters $\lambda_{N}$ and $r$ are related as

$$
\frac{r}{\lambda_{N}}=\sqrt{\frac{N}{2}\left(\frac{N}{2}+1\right)} .
$$

The algebra $\mathcal{S}_{N}^{2}$ generated by the $\hat{x}_{i}$ is clearly the simple matrix algebra $\operatorname{Mat}(N+1)$. Under the adjoint action of $S U(2)$, it decomposes into the direct $\operatorname{sum}(1) \oplus(3) \oplus(5) \oplus \ldots \oplus(2 N+1)$ of irreducible representations of $S O(3)$ 16. 


\subsection{The $q$-deformed fuzzy sphere}

The fuzzy sphere $\mathcal{S}_{N}^{2}$ is invariant under the action of $S O(3)$, or equivalently under the action of $U(s o(3))$. We shall define finite algebras $\mathcal{S}_{q, N}^{2}$ generated by $x_{i}$ for $i=1,0,-1$, which have completely analogous properties to those of $\mathcal{S}_{N}^{2}$, but which are covariant under the quantized universal enveloping algebra $U_{q}(s u(2))$. This will be done for both $q \in \mathbb{R}$ and $q$ a phase, including the appropriate reality structure. In the first case, the $\mathcal{S}_{q, N}^{2}$ will turn out to be the "discrete series" of Podleś' quantum spheres [29]. Here we will study them more closely

from the above point of view. However, we also allow $q$ to be a root of unity, with certain restrictions. In a twisted form, this case does appear naturally on $D$-branes in the $S U(2)$ WZW model, as was shown in [1].

In order to make the analogy to the undeformed case obvious, we shall perform a $q^{-}$ deformed Jordan-Wigner construction, which is covariant under $U_{q}(s u(2))$. To fix the notation, we recall the basic relations of $U_{q}(s u(2))$

$$
\begin{aligned}
{\left[H, X^{ \pm}\right] } & = \pm 2 X^{ \pm} \\
{\left[X^{+}, X^{-}\right] } & =\frac{q^{H}-q^{-H}}{q-q^{-1}}=[H]_{q}
\end{aligned}
$$

where the $q$-numbers are defined as $[n]_{q}=\frac{q^{n}-q^{-n}}{q-q^{-1}}$. The action of $U_{q}(s u(2))$ on a tensor product of representations is encoded in the coproduct

$$
\begin{aligned}
\Delta(H) & =H \otimes 1+1 \otimes H \\
\Delta\left(X^{ \pm}\right) & =X^{ \pm} \otimes q^{-H / 2}+q^{H / 2} \otimes X^{ \pm} .
\end{aligned}
$$

The antipode and the counit are given by

$$
\begin{aligned}
& S(H)=-H, \quad S\left(X^{+}\right)=-q^{-1} X^{+}, \quad S\left(X^{-}\right)=-q X^{-}, \\
& \varepsilon(H)=\varepsilon\left(X^{ \pm}\right)=0 .
\end{aligned}
$$

The star structure is related to the Cartan-Weyl involution $\theta\left(X^{ \pm}\right)=X^{\mp}, \theta(H)=H$, and will be discussed below. All symbols will now be understood to carry a label " $q$ ", which we shall omit.

An algebra $\mathcal{A}$ is called an $U_{q}(s u(2))$-module algebra if there exists an action

$$
\begin{aligned}
U_{q}(s u(2)) \times \mathcal{A} & \rightarrow \mathcal{A}, \\
(u, a) & \mapsto u \triangleright a
\end{aligned}
$$

\footnotetext{
${ }^{4}$ We use the opposite coproduct than in the standard conventions, but nevertheless the invariant tensors and $\hat{R}$-matrices will be the standard ones. The reason for this is explained in Appendix A.
} 
which satisfies $u \triangleright(a b)=\left(u_{(1)} \triangleright a\right)\left(u_{(2)} \triangleright b\right)$ for $a, b \in \mathcal{A}$. Here $\Delta(u)=u_{(1)} \otimes u_{(2)}$ is the Sweedler notation for the coproduct.

Consider $q$-deformed creation and anihilation operators $A_{\alpha}, A^{+\alpha}$ for $\alpha= \pm \frac{1}{2}$, which satisfy the relations (cp. 36])

$$
\begin{aligned}
A^{+\alpha} A_{\beta} & =\delta_{\beta}^{\alpha}+q \hat{R}_{\beta \delta}^{\alpha \gamma} A_{\gamma} A^{+\delta} \\
\left(P^{-}\right)_{\gamma \delta}^{\alpha \beta} A_{\alpha} A_{\beta} & =0 \\
\left(P^{-}\right)_{\gamma \delta}^{\alpha \beta} A^{+\delta} A^{+\gamma} & =0
\end{aligned}
$$

where $\hat{R}_{\beta \delta}^{\alpha \gamma}=q\left(P^{+}\right)_{\beta \delta}^{\alpha \gamma}-q^{-1}\left(P^{-}\right)_{\beta \delta}^{\alpha \gamma}$ is the decomposition of the $\hat{R}$-matrix of $U_{q}(s u(2))$ into the projection operators on the symmetric and antisymmetric part. They can be written as

$$
\begin{aligned}
\left(P^{-}\right)_{\gamma \delta}^{\alpha \beta} & =\frac{1}{-[2]_{q}} \varepsilon^{\alpha \beta} \varepsilon_{\gamma \delta}, \\
\left(P^{+}\right)_{\gamma \delta}^{\alpha \beta} & =\sigma_{i}^{\alpha \beta} \sigma_{\gamma \delta}^{i}
\end{aligned}
$$

Here $\varepsilon_{\alpha \beta}$ is the $q$-deformed invariant antisymmetric tensor, and $\sigma_{\alpha \beta}^{i}$ are the $q$-deformed Clebsch-Gordan coefficients; they are given explicitly in the Appendix. The factor $-[2]_{q}^{-1}$ arises from the relation $\varepsilon^{\alpha \beta} \varepsilon_{\alpha \beta}=-[2]_{q}$. The above relations are covariant under $U_{q}(s u(2))$, and define a left $U_{q}(s u(2))$-module algebra. We shall denote the action on the generators with lower indices by

$$
u \triangleright A_{\alpha}=A_{\beta} \pi_{\alpha}^{\beta}(u),
$$

so that $\pi_{\beta}^{\alpha}(u v)=\pi_{\gamma}^{\alpha}(u) \pi_{\beta}^{\gamma}(v)$ for $u, v \in U_{q}(s u(2))$. The generators with upper indices transform in the contragredient representation, which means that

$$
A_{\alpha}^{+}:=\varepsilon_{\alpha \beta} A^{+\beta}
$$

transforms in the same way under $U_{q}(s u(2))$ as $A_{\alpha}$.

We consider again the corresponding Fock space $\mathcal{F}$ generated by the $A^{+^{\alpha}}$ acting on the vacuum $|0\rangle$, and its sectors

$$
\mathcal{F}_{N}=\left\{\sum A^{+\alpha_{1}} \ldots A^{+\alpha_{N}}|0\rangle(N \text { creation operators })\right\} .
$$

It is well-known that these subspaces $\mathcal{F}_{N}$ are $N+1$-dimensional, as they are when $q=1$, and it follows that they form irreducible representations of $U_{q}(s u(2)$ ) (at root of unity, this will be true due to the restriction (2.36) we shall impose). This will be indicated by writing $\mathcal{F}_{N}=(N+1)$, and the decomposition of $\mathcal{F}$ into irreducible representations is

$$
\mathcal{F}=\mathcal{F}_{0} \oplus \mathcal{F}_{1} \oplus \mathcal{F}_{2} \oplus \ldots=(1) \oplus(2) \oplus(3) \oplus \ldots
$$


Now we define

$$
\hat{Z}_{i}=A^{+\alpha^{\prime}} \varepsilon_{\alpha \alpha^{\prime}} \sigma_{i}^{\alpha \beta} A_{\beta}
$$

and

$$
\hat{N}=\sum_{\alpha} A^{+\alpha^{\prime}} \varepsilon_{\alpha \alpha^{\prime}} \varepsilon^{\alpha \beta} A_{\beta}
$$

After some calculations, these operators can be shown to satisfy the relations

$$
\begin{aligned}
& \varepsilon_{k}^{i j} \hat{Z}_{i} \hat{Z}_{j}=\frac{q^{-1}}{\sqrt{[2]_{q}}}\left(q^{-1}[2]_{q}-\lambda \hat{N}\right) \hat{Z}_{k} \\
& \hat{Z}^{2}:=g^{i j} \hat{Z}_{i} \hat{Z}_{j}=q^{-2} \frac{[2]_{q}+\hat{N}}{[2]_{q}} \hat{N}
\end{aligned}
$$

Here $\lambda=\left(q-q^{-1}\right), g^{i j}$ is the $q$-deformed invariant tensor for spin 1 representations, and $\varepsilon_{k}^{i j}$ is the corresponding $q$-deformed Clebsch-Gordan coefficient; they are given in the Appendix. Moreover, one can verify that

$$
\begin{aligned}
\hat{N} A^{+\alpha} & =q^{-3} A^{+\alpha}+q^{-2} A^{+\alpha} \hat{N} \\
\hat{N} A_{\alpha} & =-q^{-1} A_{\alpha}+q^{2} A_{\alpha} \hat{N},
\end{aligned}
$$

which implies that

$$
\left[\hat{N}, \hat{Z}_{i}\right]=0
$$

On the subspace $\mathcal{F}_{N}$, the "number" operator $\hat{N}$ takes the value

$$
\hat{N} \mathcal{F}_{N}=q^{-N-2}[N]_{q} \mathcal{F}_{N}
$$

It is convenient to introduce also an undeformed number operator $\hat{n}$ which has eigenvalues

$$
\hat{n} \mathcal{F}_{N}=N \mathcal{F}_{N}
$$

in particular $\hat{n} A_{\alpha}=A_{\alpha}(\hat{n}-1)$.

On the subspaces $\mathcal{F}_{N}$, the relations $(2.20)$ become

$$
\begin{aligned}
\varepsilon_{k}^{i j} x_{i} x_{j} & =\Lambda_{N} x_{k}, \\
x \cdot x & :=g^{i j} x_{i} x_{j}=r^{2} .
\end{aligned}
$$

Here the variables have been rescaled to $x_{i}$ with

$$
x_{i}=r \frac{q^{\hat{n}+2}}{\sqrt{[2]_{q} C_{N}}} \hat{Z}_{i} .
$$


The $r$ is a real number, and we have defined

$$
\begin{aligned}
C_{N} & =\frac{[N]_{q}[N+2]_{q}}{[2]_{q}^{2}}, \\
\Lambda_{N} & =r \frac{[2]_{q^{N+1}}}{\sqrt{[N]_{q}[N+2]_{q}}} .
\end{aligned}
$$

Using a completeness relation (see Appendix A), (2.24) can equivalently be written as

$$
\left(P^{-}\right)_{k l}^{i j} x_{i} x_{j}=\frac{1}{[2]_{q^{2}}} \Lambda_{N} \varepsilon_{k l}^{n} x_{n} .
$$

There is no $i$ in the commutation relations, because we use a weight basis instead of Cartesian coordinates. One can check that these relations precisely reproduce the "discrete" series of Podleś' quantum spheres (after another rescaling), see [29], Proposition 4.II. Hence we define $\mathcal{S}_{q, N}^{2}$ to be the algebra generated by the variables $x_{i}$ acting on $\mathcal{F}_{N}$. Equipped with a suitable star structure and a differential structure, this will be the $q$-deformed fuzzy sphere.

It is easy to see that the algebra $\mathcal{S}_{q, N}^{2}$ is simply the full matrix algebra $\operatorname{Mat}(N+1)$, i.e. it is the same algebra as $\mathcal{S}_{N}^{2}$ for $q=1$. This is because $\mathcal{F}_{N}$ is an irreducible representation of $U_{q}(s u(2))$. To see it, we use complete reducibility [32] of the space of polynomials in $x_{i}$ of degree $\leq k$ to conclude that it decomposes into the direct sum of irreducible representations $(1) \oplus(3) \oplus(5) \oplus \ldots \oplus(2 k+1)$. Counting dimensions and noting that $x_{1}^{N} \neq 0 \in(2 N+1)$, it follows that $\operatorname{dim}\left(\mathcal{S}_{q, N}^{2}\right)=(N+1)^{2}=\operatorname{dim} \operatorname{Mat}(N+1)$, and hence

$$
\mathcal{S}_{q, N}^{2}=(1) \oplus(3) \oplus(5) \oplus \ldots \oplus(2 N+1) .
$$

This is true even if $q$ is a root of unity provided (2.36) below holds, a relation which will be necessary for other reasons as well. This is the decomposition of the functions on the $q^{-}$ deformed fuzzy sphere into $q$-spherical harmonics, and it is automatically truncated. Note however that not all information about a (quantum) space is encoded in its algebra of functions; in addition, one must specify for example a differential calculus and symmetries. For example, the action of $U_{q}(s u(2))$ on $\mathcal{S}_{q, N}^{2}$ is different from the action of $U(s u(2))$ on $\mathcal{S}_{N}^{2}$.

The covariance of $\mathcal{S}_{q, N}^{2}$ under $U_{q}(s u(2))$ can also be stated in terms of the quantum adjoint action. It is convenient to consider the cross-product algebra $U_{q}(s u(2)) \ltimes \mathcal{S}_{q, N}^{2}$, which as a vector space is equal to $U_{q}(s u(2)) \otimes \mathcal{S}_{q, N}^{2}$, equipped with an algebra structure defined by

$$
u x=\left(u_{(1)} \triangleright x\right) u_{(2)} .
$$

Here the $\triangleright$ denotes the action of $u \in U_{q}(s u(2))$ on $x \in \mathcal{S}_{q, N}^{2}$. Conversely, the action of $U_{q}(s u(2))$ on $\mathcal{S}_{q, N}^{2}$ can be written as $u \triangleright x=u_{(1)} x S u_{(2)}$. The relations (2.29) of $U_{q}(s u(2)) \ltimes \mathcal{S}_{q, N}^{2}$ are automatically realized on the representation $\mathcal{F}_{N}$. 
Since both algebras $\mathcal{S}_{q, N}^{2}$ and $U_{q}(s u(2))$ act on $\mathcal{F}_{N}$ and generate the full matrix algebra $\operatorname{Mat}(N+1)$, it must be possible to express the generators of $U_{q}(s u(2))$ in terms of the $\hat{Z}_{i}$. The explicit relation can be obtained by comparing the relations (2.24) with (2.29). One finds

$$
\begin{aligned}
X^{+} q^{-H / 2} & =q^{N+3} \hat{Z}_{1}, \\
X^{-} q^{-H / 2} & =-q^{N+1} \hat{Z}_{-1}, \\
q^{-H} & =\frac{[2]_{q^{N+1}}}{[2]_{q}}+\frac{q^{N+2}\left(q-q^{-1}\right)}{\sqrt{[2]_{q}}} \hat{Z}_{0},
\end{aligned}
$$

if acting on $\mathcal{F}_{N}$. In fact, this defines an algebra map

$$
j: \quad U_{q}(s u(2)) \rightarrow \mathcal{S}_{q, N}^{2}
$$

which satisfies

$$
j\left(u_{(1)}\right) x j\left(S u_{(2)}\right)=u \triangleright x
$$

for $x \in \mathcal{S}_{q, N}^{2}$ and $u \in U_{q}(s u(2))$. This is analogous to results in [5, 6]. We shall often omit $j$ from now on. In particular, $\mathcal{S}_{q, N}^{2}$ is the quotient of the algebra $U_{q}(s u(2))$ by the relation (2.25) $(2.20)$. Moreover, one can verify that it is represented correctly on $\mathcal{F}_{N}$ by observing that $X^{+}\left(A_{1 / 2}^{+}\right)^{N}|0\rangle=0$, which means that $\left(A_{1 / 2}^{+}\right)^{N}|0\rangle$ is the highest-weight vector of $\mathcal{F}_{N}$.

\subsection{Reality structure for $q \in \mathbb{R}$}

In order to define a real quantum space, we must also construct a star structure, which is an involutive anti-linear anti-algebra map. For real $q$, the algebra (2.12) is consistent with the following star structure

$$
\begin{aligned}
\left(A_{\alpha}\right)^{*} & =A^{+\alpha} \\
\left(A^{+\alpha}\right)^{*} & =A_{\alpha}
\end{aligned}
$$

This can be verified using the standard compatibility relations of the $\hat{R}$-matrix with the invariant tensor [14]. On the generators $x_{i}$, it implies the relation

$$
x_{i}^{*}=g^{i j} x_{j}
$$

\footnotetext{
${ }^{5}$ more precisely, its finite-dimensional representation.
} 
as well as the equality

$$
\hat{N}^{*}=\hat{N}
$$

The algebras $\mathcal{S}_{q, N}^{2}$ are now precisely Podleś' "discrete" $C^{*}$ algebras $\tilde{S}_{q, c(N+1)}^{2}$. Using (2.30), this is equivalent to

$$
H^{*}=H,\left(X^{ \pm}\right)^{*}=X^{\mp},
$$

which is the star-structure for the compact form $U_{q}(s u(2))$. It is well-known that there is a unique Hilbert space structure on the subspaces $\mathcal{F}_{N}$ such that they are unitary irreducible representations of $U_{q}(s u(2))$. Then the above star is simply the operator adjoint.

\subsection{Reality structure for $q$ a phase}

When $q$ is a phase, finding the correct star structure is not quite so easy. The difference with the case $q \in \mathbb{R}$ is that $\Delta\left(u^{*}\right)=(* \otimes *) \Delta^{\prime}(u)$ for $|q|=1$ and $u \in U_{q}(s u(2))$, where $\Delta^{\prime}$ denotes the flipped coproduct. We shall define a star only on the algebra $\mathcal{S}_{q, N}^{2}$ generated by the $x_{i}$, and not on the full algebra generated by $A_{\alpha}$ and $A_{\alpha}^{+}$.

There appears to be an obvious choice at first sight, namely $x_{i}^{*}=x_{i}$, which is indeed consistent with (2.24). However, it is the wrong choice for our purpose, because it induces the noncompact star structure $U_{q}(s l(2, \mathbb{R}))$.

Instead, we define a star-structure on $\mathcal{S}_{q, N}^{2}$ as follows. The algebra $U_{q}(s u(2))$ acts on the space $\mathcal{S}_{q, N}^{2}$, which generically decomposes as $(1) \oplus(3) \oplus \ldots \oplus(2 N+1)$. This decomposition should be a direct sum of unitary representations of the compact form of $U_{q}(s u(2))$, which means that the star structure on $U_{q}(s u(2))$ should be (2.35), as it is for real $q$. There is a slight complication, because not all finite-dimensional irreducible representations are unitary if $q$ is a phase [20]. However, all representations with dimension $\leq 2 N+1$ are unitary provided $q$ has the form

$$
q=e^{i \pi \varphi}, \quad \text { with } \varphi<\frac{1}{2 N} .
$$

This will be assumed from now on.

As was pointed out before, we can consider the algebra $\mathcal{S}_{q, N}^{2}$ as a quotient of $U_{q}(s u(2))$ via (2.30). It acts on $\mathcal{F}_{N}$, which is an irreducible representation of $U_{q}(s u(2))$, and hence has a natural Hilbert space structure. We define the star on the operator algebra $\mathcal{S}_{q, N}^{2}$ by the adjoint (that is by the matrix adjoint in an orthonormal basis), hence by the star (2.35) using the identification (2.30).

There is a very convenient way to write down this star structure on the generators $x_{i}$, similar as in [33. It involves an element $\omega$ of an extension of $U_{q}(s u(2))$ introduced by 21] 
and [23], which implements the Weyl reflection on irreducible representations. The essential properties are

$$
\begin{aligned}
\Delta(\omega) & =\mathcal{R}^{-1} \omega \otimes \omega, \\
\omega u \omega^{-1} & =\theta S^{-1}(u), \\
\omega^{2} & =v \epsilon,
\end{aligned}
$$

where $v$ and $\epsilon$ are central elements in $\in U_{q}(s u(2))$ which take the values $q^{-N(N+2) / 2}$ resp. $(-1)^{N}$ on $\mathcal{F}_{N}$. Here $\mathcal{R}=\mathcal{R}_{1} \otimes \mathcal{R}_{2} \in U_{q}(s u(2)) \otimes U_{q}(s u(2))$ is the universal $R$ element. In a suitable (weight) basis of a unitary representation of $U_{q}(s u(2))$, the matrix representing $\omega$ is given the invariant tensor, $\pi_{j}^{i}(\omega)=-q^{-N(N+2) / 4} g^{i j}$, and $\omega^{*}=\omega^{-1}$. This is discussed in detail in [33]. From now on, we denote with $\omega$ the element in $\mathcal{S}_{q, N}^{2}$ which represents this element on $\mathcal{F}_{N}$.

We claim that the star structure on $\mathcal{S}_{q, N}^{2}$ as explained above is given by the following formula:

$$
x_{i}^{*}=-\omega x_{i} \omega^{-1}=x_{j} L_{k}^{-j} q^{-2} g^{k i},
$$

where

$$
L^{-i}=\pi_{j}^{i}\left(\mathcal{R}_{1}^{-1}\right) \mathcal{R}_{2}^{-1}
$$

as usual [14]; a priori, $L^{-i}{ }_{i} \in U_{q}(s u(2))$, but it is understood here as an element of $\mathcal{S}_{q, N}^{2}$ via (2.30). One can easily verify using $\left(\varepsilon_{k}^{i j}\right)^{*}=-\varepsilon_{k}^{j i}$ (for $|q|=1$ ) that (2.40) is consistent with the relations (2.24) and (2.25). In the limit $q \rightarrow 1, L_{j}^{-i} \rightarrow \delta_{j}^{i}$, therefore (2.40) agrees with (2.34) in the classical limit. Hence we define the $q$-deformed fuzzy sphere for $q$ a phase to be the algebra $\mathcal{S}_{q, N}^{2}$ equipped with the star-structure (2.40).

To show that (2.40) is correct in the sense explained above, it is enough to verify that it induces the star structure (2.35) on $U_{q}(s u(2))$, since both $U_{q}(s u(2))$ and $\mathcal{S}_{q, N}^{2}$ generate the same algebra $\operatorname{Mat}(N+1)$. This can easily be seen using (2.38) and (2.30). A somewhat related conjugation has been proposed in [33, 24] using the universal element $\mathcal{R}$.

\subsection{Invariant integral}

The integral on $\mathcal{S}_{q, N}^{2}$ is defined to be the unique functional on $\mathcal{S}_{q, N}^{2}$ which is invariant under the (quantum adjoint) action of $U_{q}(s u(2))$. It is given by the projection on the trivial sector in the decomposition (2.28). We claim that it can be written explicitly using the quantum trace:

$$
\int_{\mathcal{S}_{q, N}^{2}} f\left(x_{i}\right):=4 \pi r^{2} \frac{1}{[N+1]_{q}} \operatorname{Tr}_{q}\left(f\left(x_{i}\right)\right)=4 \pi r^{2} \frac{1}{[N+1]_{q}} \operatorname{Tr}\left(f\left(x_{i}\right) q^{-H}\right)
$$


for $f\left(x_{i}\right) \in \mathcal{S}_{q, N}^{2}$, where the trace is taken on $\mathcal{F}_{N}$. Using $S^{-2}(u)=q^{H} u q^{-H}$ for $u \in U_{q}(s u(2))$, it follows that

$$
\int_{\mathcal{S}_{q, N}^{2}} f g=\int_{\mathcal{S}_{q, N}^{2}} S^{-2}(g) f .
$$

This means that it is indeed invariant under the quantum adjoint action,

$$
\begin{aligned}
\int_{\mathcal{S}_{q, N}^{2}} u \triangleright f\left(x_{i}\right) & =\int_{\mathcal{S}_{q, N}^{2}} u_{1} f\left(x_{i}\right) S\left(u_{2}\right) \\
& =\int_{\mathcal{S}_{q, N}^{2}} S^{-1}\left(u_{2}\right) u_{1} f\left(x_{i}\right)=\varepsilon(u) \int_{\mathcal{S}_{q, N}^{2}} f\left(x_{i}\right),
\end{aligned}
$$

using the identification (2.30). The normalization constant is obtained from

$$
\operatorname{Tr}_{q}(1)=\operatorname{Tr}\left(q^{-H}\right)=q^{N}+q^{N-2}+\ldots+q^{-N}=[N+1]_{q}
$$

on $\mathcal{F}_{N}$, so that $\int_{\mathcal{S}_{q, N}^{2}} 1=4 \pi r^{2}$.

Lemma 2.1 Let $f \in \mathcal{S}_{q, N}^{2}$. Then

$$
\left(\int_{\mathcal{S}_{q, N}^{2}} f\right)^{*}=\int_{\mathcal{S}_{q, N}^{2}} f^{*}
$$

for real $q$, and

$$
\left(\int_{\mathcal{S}_{q, N}^{2}} f\right)^{*}=\int_{\mathcal{S}_{q, N}^{2}} f^{*} q^{2 H}
$$

for $q$ a phase, with the appropriate star structure (2.34) respectively (2.49). In (2.46), we use (2.30.

Proof Assume first that $q$ is real, and consider the functional

$$
I_{q, N}(f):=\operatorname{Tr}\left(f^{*} q^{-H}\right)^{*}
$$

for $f \in \mathcal{S}_{q, N}^{2}$. Then

$$
\begin{aligned}
I_{q, N}(u \triangleright f) & =\operatorname{Tr}\left(\left(u_{1} f S\left(u_{2}\right)\right)^{*} q^{-H}\right)^{*} \\
& =\operatorname{Tr}\left(S^{-1}\left(\left(u^{*}\right)_{2}\right) f^{*}\left(u^{*}\right)_{1} q^{-H}\right)^{*}=\operatorname{Tr}\left(f^{*}\left(u^{*}\right)_{1} S\left(\left(u^{*}\right)_{2}\right) q^{-H}\right)^{*} \\
& =\varepsilon(u) I_{q, N}(f),
\end{aligned}
$$


where $(S(u))^{*}=S^{-1}\left(u^{*}\right)$ and $(* \otimes *) \Delta(u)=\Delta\left(u^{*}\right)$ was used. Hence $I_{q, N}(f)$ is invariant as well, and (2.45) follows using uniqueness of the integral (up to normalization). For $|q|=1$, we define

$$
\tilde{I}_{q, N}(f):=\operatorname{Tr}\left(f^{*} q^{H}\right)^{*}
$$

with the star structure (2.40). Using $(S(u))^{*}=S\left(u^{*}\right)$ and $(* \otimes *) \Delta(u)=\Delta^{\prime}\left(u^{*}\right)$, an analogous calculation shows that $\tilde{I}_{q, N}$ is invariant under the action of $U_{q}(s u(2))$, which again implies (2.46).

For $|q|=1$, the integral is neither real nor positive, hence it cannot be used for a GNS construction. Nevertheless, it is clearly the appropriate functional to define an action for field theory, since it is invariant under $U_{q}(s u(2))$. To find a way out, we introduce an auxiliary antilinear algebra-map on $\mathcal{S}_{q, N}^{2}$ by

$$
\bar{f}=S^{-1}\left(f^{*}\right)
$$

where $S$ is the antipode on $U_{q}(s u(2))$, using (2.30). Note that $S$ preserves the relation (2.25), hence it is well-defined on $\mathcal{S}_{q, N}^{2}$. This is not a star structure, since

$$
\overline{\bar{f}}=S^{-2} f
$$

for $|q|=1$. Using (2.30), one finds in particular

$$
\overline{x_{i}}=-g^{i j} x_{j}
$$

This is clearly consistent with the relations (2.24) and (2.25). We claim that (2.46) can now be stated as

$$
\left(\int_{\mathcal{S}_{q, N}^{2}} f\right)^{*}=\int_{\mathcal{S}_{q, N}^{2}} \bar{f} \quad \text { for }|q|=1 .
$$

To see this, observe first that

$$
\operatorname{Tr}(S(f))=\operatorname{Tr}(f),
$$

which follows either from the fact that $\hat{I}_{q, N}(f):=\operatorname{Tr}\left(S^{-1}(f) q^{H}\right)=\operatorname{Tr}\left(S^{-1}\left(q^{-H} f\right)\right)$ is yet another invariant functional, or using $\omega f \omega^{-1}=\theta S^{-1}(f)$ together with the observation that the matrix representations of $X^{ \pm}$in a suitable basis are real. This implies

$$
\operatorname{Tr}_{q}\left(f^{*} q^{2 H}\right)=\operatorname{Tr}\left(f^{*} q^{H}\right)=\operatorname{Tr}\left(S\left(q^{-H} S^{-1}\left(f^{*}\right)\right)\right)=\operatorname{Tr}\left(q^{-H} S^{-1}\left(f^{*}\right)\right)=\operatorname{Tr}_{q}(\bar{f}),
$$

and (2.50) follows. Now we can write down a positive inner product on $\mathcal{S}_{q, N}^{2}$ : 
Lemma 2.2 The sesquilinear forms

$$
(f, g):=\int_{\mathcal{S}_{q, N}^{2}} f^{*} g \quad \text { for } q \in \mathbb{R}
$$

and

$$
(f, g):=\int_{\mathcal{S}_{q, N}^{2}} \bar{f} g, \quad \text { for }|q|=1
$$

are hermitian, that is $(f, g)^{*}=(g, f)$, and satisfy

$$
(f, u \triangleright g)=\left(u^{*} \triangleright f, g\right)
$$

for both $q \in \mathbb{R}$ and $|q|=1$. They are positive definite provided (2.36) holds for $|q|=1$, and define a Hilbert space structure on $\mathcal{S}_{q, N}^{2}$.

Proof For $q \in \mathbb{R}$, we have

$$
\begin{aligned}
(f, u \triangleright g) & =\int_{\mathcal{S}_{q, N}^{2}} f^{*} u_{1} g S u_{2}=\int_{\mathcal{S}_{q, N}^{2}} S^{-1}\left(u_{2}\right) f^{*} u_{1} g=\int_{\mathcal{S}_{q, N}^{2}}\left(S\left(\left(u^{*}\right)_{2}\right)^{*} f^{*}\left(\left(u^{*}\right)_{1}\right)^{*} g\right. \\
& =\int_{\mathcal{S}_{q, N}^{2}}\left(\left(u^{*}\right)_{1} f S\left(u^{*}\right)_{2}\right)^{*} g=\left(u^{*} \triangleright f, g\right),
\end{aligned}
$$

and hermiticity is immediate. For $|q|=1$, consider

$$
\begin{aligned}
(f, u \triangleright g) & =\int_{\mathcal{S}_{q, N}^{2}} \bar{f} u_{1} g S u_{2}=\int_{\mathcal{S}_{q, N}^{2}} S^{-1}\left(u_{2}\right) S^{-1}\left(f^{*}\right) u_{1} g=\int_{\mathcal{S}_{q, N}^{2}} S^{-1}\left(\left(u^{*}\right)_{1} f S\left(u^{*}\right)_{2}\right)^{*} g \\
& =\left(u^{*} \triangleright f, g\right) .
\end{aligned}
$$

Hermiticity follows using (2.50):

$$
(f, g)^{*}=\int_{\mathcal{S}_{q, N}^{2}} \overline{\bar{f}} \bar{g}=\int_{\mathcal{S}_{q, N}^{2}} S^{-2}(f) \bar{g}=\int_{\mathcal{S}_{q, N}^{2}} \bar{g} f=(g, f) .
$$

Using the assumption (2.36) for $|q|=1$, it is not difficult to see that they are also positivedefinite. 


\section{Differential Calculus}

In order to write Lagrangians, it is convenient to use the notion of an (exterior) differential calculus [37, 8]. A covariant differential calculus over $\mathcal{S}_{q, N}^{2}$ is a graded bimodule $\Omega_{q, N}^{*}=$ $\oplus_{n} \Omega_{q, N}^{n}$ over $\mathcal{S}_{q, N}^{2}$ which is a $U_{q}(s u(2))$-module algebra, together with an exterior derivative $d$ which satisfies $d^{2}=0$ and the graded Leibnitz rule. We define the dimension of a calculus to be the rank of $\Omega_{q, N}^{1}$ as a free right $\mathcal{S}_{q, N}^{2}$-module.

\subsection{First-order differential forms}

Differential calculi for the Podleś sphere have been studied before [30, 4]. It turns out that 2-dimensional calculi do not exist for the cases we are interested in; however there exists a unique 3-dimensional module of 1-forms. As opposed to the classical case, it contains an additional "radial" one-form. This will lead to an additional scalar field, which will be discussed later.

By definition, it must be possible to write any term $x_{i} d x_{j}$ in the form $\sum_{k} d x_{k} f_{k}(x)$. Unfortunately the structure of the module of 1-forms turn out to be not quadratic, rather the $f_{k}(x)$ are polynomials of order up to 3 . In order to make it more easily tractable and to find suitable reality structures, we will construct this calculus using a different basis. First, we will define the bimodule of 1 -forms $\Omega_{q, N}^{1}$ over $\mathcal{S}_{q, N}^{2}$ which is covariant under $U_{q}(s u(2))$, such that $\left\{d x_{i}\right\}_{i}$ is a free right $\mathcal{S}_{q, N}^{2}$-module basis, together with a map $d: \mathcal{S}_{q, N}^{2} \rightarrow \Omega_{q, N}^{1}$ which satisfies the Leibnitz rule. Higher-order differential forms will be discussed below.

Consider a basis of one-forms $\xi_{i}$ for $i=-1,0,1$ with the covariant commutation relations

$$
x_{i} \xi_{j}=\hat{R}_{i j}^{k l} \xi_{k} x_{l}
$$

using the $(3) \otimes(3) \hat{R}$-matrix of $U_{q}(s u(2))$. It has the projector decomposition

$$
\hat{R}_{i j}^{k l}=q^{2}\left(P^{+}\right)_{i j}^{k l}-q^{-2}\left(P^{-}\right)_{i j}^{k l}+q^{-4}\left(P^{0}\right)_{i j}^{k l},
$$

where $\left(P^{0}\right)_{i j}^{k l}=\frac{1}{[3]_{q}} g^{k l} g_{i j}$, and $\left(P^{-}\right)_{i j}^{k l}=\sum_{n} \frac{1}{[2]_{q^{2}}} \varepsilon_{n}^{k l} \varepsilon_{i j}^{n}$. The relations (3.1) are consistent with (2.25) and (2.24), using the braiding relations 31]

$$
\begin{aligned}
\hat{R}_{i j}^{k l} \hat{R}_{l u}^{r s} \varepsilon_{n}^{j u} & =\varepsilon_{t}^{k r} \hat{R}_{i n}^{t s}, \\
\hat{R}_{i j}^{k l} \hat{R}_{l u}^{r s} g^{j u} & =g^{k r} \delta_{i}^{s}
\end{aligned}
$$

\footnotetext{
${ }^{6}$ they are not equivalent to $u \xi_{i}=u_{(1)} \triangleright \xi_{i} u_{(2)}$.
} 
and the quantum Yang-Baxter equation $\hat{R}_{12} \hat{R}_{23} \hat{R}_{12}=\hat{R}_{23} \hat{R}_{12} \hat{R}_{23}$, in shorthand-notation [14. We define $\Omega_{q, N}^{1}$ to be the free right module over $\mathcal{S}_{q, N}^{2}$ generated by the $\xi_{i}$. It is clearly a bimodule over $\mathcal{S}_{q, N}^{2}$. To define the exterior derivative, consider

$$
\Theta:=x \cdot \xi=x_{i} \xi_{j} g^{i j},
$$

which is a singlet under $U_{q}(s u(2))$. It turns out (see Appendix B) that $\left[\Theta, x_{i}\right] \neq 0 \in \Omega_{q, N}^{1}$. Hence

$$
d f:=[\Theta, f(x)]
$$

defines a nontrivial derivation $d: \mathcal{S}_{q, N}^{2} \rightarrow \Omega_{q, N}^{1}$, which completes the definition of the calculus up to first order. In particular, it is shown in Appendix B that

$$
d x_{i}=-\Lambda_{N} \varepsilon_{i}^{n k} x_{n} \xi_{k}+\left(q-q^{-1}\right)\left(q x_{i} \Theta-r^{2} q^{-1} \xi_{i}\right) .
$$

Since all terms are linearly independent, this is a 3-dimensional first-order differential calculus, and by the uniqueness it agrees with the 3-dimensional calculus in [30, 4. In view of (3.7), it is not surprising that the commutation relations between the generators $x_{i}$ and $d x_{i}$ are very complicated [4]; will not write them down here. The meaning of the $\xi$-forms will become more clear in Section 3.4.

Using (7.5) and the relation $\xi \cdot x=q^{4} x \cdot \xi$, one finds that

$$
x \cdot d x=\left(-\Lambda_{N}^{2}+\left([2]_{q^{2}}-2\right) r^{2}\right) \Theta .
$$

On the other hand, this must be equal to $x_{i} \Theta x_{j} g^{i j}-r^{2} \Theta$, which implies that

$$
x_{i} \Theta x_{j} g^{i j}=\alpha r^{2} \Theta
$$

with

$$
\alpha=[2]_{q^{2}}-1-\frac{\Lambda_{N}^{2}}{r^{2}}=1-\frac{1}{C_{N}} .
$$

Combining this, it follows that

$$
d x \cdot x=r^{2} \frac{1}{C_{N}} \Theta=-x \cdot d x .
$$

Moreover, using the identity (7.7) one finds

$$
\varepsilon_{i}^{j k} x_{j} d x_{k}=\left(\alpha-q^{2}\right) r^{2} \varepsilon_{i}^{j k} x_{j} \xi_{k}-\Lambda_{N} r^{2} \xi_{i}+q^{2} \Lambda_{N} x_{i} \Theta,
$$

which together with (3.7) yields

$$
\xi_{i}=\frac{q^{2}}{r^{2}} \Theta x_{i}+\frac{q^{2} C_{N} \Lambda_{N}}{r^{4}} \varepsilon_{i}^{j k} x_{j} d x_{k}-q^{2}\left(1-q^{2}\right) \frac{C_{N}}{r^{2}} d x_{i} .
$$




\subsection{Higher-order differential forms}

Podleś [30] has constructed an extension of the above 3-dimensional calculus including higher-order forms for a large class of quantum spheres. This class does not include ours, however, hence we will give a different construction based on $\xi$-variables, which will be suitable for $q$ a phase as well.

Consider the algebra

$$
\xi_{i} \xi_{j}=-q^{2} \hat{R}_{i j}^{k l} \xi_{k} \xi_{l}
$$

which is equivalent to $\left(P^{+}\right)_{k l}^{i j} \xi_{i} \xi_{j}=0,\left(P^{0}\right)_{k l}^{i j} \xi_{i} \xi_{j}=0$ where $P^{+}$and $P^{0}$ are the projectors on the symmetric components of $(3) \otimes(3)$ as above; hence the product is totally $\left(q^{-}\right)$antisymmetric. It is not hard to see (and well-known) that the dimension of the space of polynomials of order $n$ in the $\xi$ is $(3,3,1)$ for $n=(1,2,3)$, and zero for $n>3$, as classically. We define $\Omega_{q, N}^{n}$ to be the free right $\mathcal{S}_{q, N}^{2}$-module with the polynomials of order $n$ in $\xi$ as basis; this is covariant under $U_{q}(s u(2))$. Then $\Omega_{q, N}^{n}$ is in fact a (covariant) $\mathcal{S}_{q, N}^{2}$-bimodule, since the commutation relations (3.1) between $x$ and $\xi$ are consistent with (3.12), which follows from the quantum Yang-Baxter equation. There remains to construct the exterior derivative. To find it, we first note that (perhaps surprisingly) $\Theta^{2} \neq 0$, rather

$$
\Theta^{2}=-\frac{q^{-2} \Lambda_{N}}{[2]_{q^{2}}} \varepsilon^{i j k} x_{i} \xi_{j} \xi_{k}
$$

The $\varepsilon^{i j k}$ is defined in (7.8). By a straightforward but lengthy calculation which is sketched in Appendix B, one can show that

$$
d x_{i} d x_{j} g^{i j}+\frac{r^{2}}{C_{N}} \Theta^{2}=0 .
$$

We will show below that an extension of the calculus to higher-order forms exists; then this can be rewritten as

$$
d \Theta-\Theta^{2}=0 .
$$

The fact that $\Theta^{2} \neq 0$ makes the construction of the extension more complicated, since now $\alpha^{(n)} \rightarrow\left[\Theta, \alpha^{(n)}\right]_{ \pm}$does not define an exterior derivative. To remedy this, the following observation is useful: the map

$$
\begin{aligned}
*_{H}: \Omega_{q, N}^{1} & \rightarrow \Omega_{q, N}^{2} \\
\xi_{i} & \mapsto-\frac{q^{-2} \Lambda_{N}}{[2]_{q^{2}}} \varepsilon_{i}^{j k} \xi_{j} \xi_{k}
\end{aligned}
$$


defines a left-and right $\mathcal{S}_{q, N}^{2}$-module map; in other words, the commutation relations between $\xi_{i}$ and $x_{j}$ are the same as between $*_{H}\left(\xi_{i}\right)$ and $x_{j}$. This follows from the braiding relation (3.3). This is in fact the natural analogue of the Hodge-star on 1-forms in our context, and will be discussed further below. Here we note the important identity

$$
\alpha\left(*_{H} \beta\right)=\left(*_{H} \alpha\right) \beta
$$

for any $\alpha, \beta \in \Omega_{q, N}^{1}$, which is proved in Appendix B. Now (3.13) can be stated as

$$
*_{H}(\Theta)=\Theta^{2}
$$

and applying $*_{H}$ to $d f=[\Theta, f(Y)]$ one obtains

$$
\left[\Theta^{2}, f(x)\right]=*_{H} d f(x) .
$$

Now we define the map

$$
\begin{aligned}
d: \Omega_{q, N}^{1} & \rightarrow \Omega_{q, N}^{2} \\
\alpha & \mapsto[\Theta, \alpha]_{+}-*_{H}(\alpha) .
\end{aligned}
$$

It is easy to see that this defines a graded derivation from $\Omega_{q, N}^{1}$ to $\Omega_{q, N}^{2}$, and the previous equation implies immediately that

$$
(d \circ d) f=0 .
$$

In particular,

$$
d \xi_{i}=\left(1-q^{2}\right) \xi \Theta+\frac{q^{-2} \Lambda_{N}}{[2]_{q^{2}}} \varepsilon_{i}^{j k} \xi_{j} \xi_{k} .
$$

To complete the differential calculus, we extend it to $\Omega_{q, N}^{3}$ by

$$
\begin{aligned}
d: \Omega_{q, N}^{2} & \rightarrow \Omega_{q, N}^{3}, \\
\alpha^{(2)} & \mapsto\left[\Theta, \alpha^{(2)}\right] .
\end{aligned}
$$

As is shown in Appendix B, this satisfies indeed

$$
(d \circ d) \alpha=0 \quad \text { for any } \alpha \in \Omega_{q, N}^{1} .
$$

It is easy to see that the map (3.22) is non-trivial. Moreover there is precisely one monomial of order 3 in the $\xi$ variables, given by

$$
\Theta^{3}=-\frac{q^{-6} \Lambda_{N} r^{2}}{[2]_{q^{2}}[3]_{q}} \varepsilon^{i j k} \xi_{i} \xi_{j} \xi_{k},
$$


which commutes with all functions on the sphere,

$$
\left[\Theta^{3}, f\right]=0
$$

for all $f \in \mathcal{S}_{q, N}^{2}$. Finally, we complete the definition of the Hodge star operator by

$$
*_{H}(1)=\Theta^{3},
$$

and by requiring that $\left(*_{H}\right)^{2}=i d$.

\subsection{Star structure}

A $*$-calculus (or a real form of $\Omega_{q, N}^{*}$ ) is a differential calculus which is a graded $*$-algebra such that the star preserves the grade, and satisfies [37]

$$
\begin{aligned}
\left(\alpha^{(n)} \alpha^{(m)}\right)^{*} & =(-1)^{n m}\left(\alpha^{(m)}\right)^{*}\left(\alpha^{(n)}\right)^{*}, \\
\left(d \alpha^{(n)}\right)^{*} & =d\left(\alpha^{(n)}\right)^{*}
\end{aligned}
$$

for $\alpha^{(n)} \in \Omega_{q, N}^{n}$; moreover, the action of $U_{q}(s u(2))$ must be compatible with the star on $U_{q}(s u(2))$. Again, we have to distinguish the cases $q \in \mathbb{R}$ and $|q|=1$.

1) $q \in \mathbb{R}$. In this case, the star structure must satisfy

$$
\left(d x_{i}\right)^{*}=g^{i j} d x_{j}, \quad x_{i}^{*}=g^{i j} x_{j}
$$

which by (2.25) implies

$$
\Theta^{*}=-\Theta \text {. }
$$

Using (3.11), it follows that

$$
\begin{aligned}
\xi_{i}^{*} & =-g^{i j} \xi_{j}+q^{2}\left(q-q^{-1}\right) \frac{[2]_{q} C_{N}}{r^{2}} g^{i j} d x_{j} \\
& =-g^{i j} \xi_{j}-q^{2}\left(q-q^{-1}\right) \frac{[2]_{q} C_{N}}{r^{2}} g^{i j}\left(\Lambda_{N} \varepsilon_{j}^{k l} x_{k} \xi_{l}-\left(q-q^{-1}\right)\left(q x_{j} \Theta-q^{-1} r^{2} \xi_{j}\right)\right) .
\end{aligned}
$$

To show that this is indeed compatible with (3.1), one needs the following identity

$$
q^{2}\left(q-q^{-1}\right) \frac{[2]_{q} C_{N}}{r^{2}}\left(d x_{i} x_{j}-\hat{R}_{i j}^{k l} x_{k} d x_{l}\right)=\left(\mathbf{1}-\left(\hat{R}^{2}\right)_{i j}^{k l}\right) \xi_{k} x_{l}
$$

which can be verified with some effort, see Appendix B. In particular, this shows that if one imposed $x_{i} \xi_{j}=\left(\hat{R}^{-1}\right)_{i j}^{k l} \xi_{k} x_{l}$ instead of (3.1), one would obtain an equivalent calculus. This is unlike in the flat case, where one has two inequivalent calculi [15, 28]. Moreover, one can show that this real form is consistent with (3.12). 
2) $|q|=1$. In view of (2.40), it is easy to see that the star structure in this case is

$$
\left(\xi_{i}\right)^{*}=q^{-4} \omega \xi_{i} \omega^{-1}, \quad x_{i}^{*}=-\omega x_{i} \omega^{-1} .
$$

Recall that $\omega$ is a particular unitary element of $\mathcal{S}_{q, N}^{2}$ introduced in Section 2.4.

It is obvious using $\left(\hat{R}_{i j}^{k l}\right)^{*}=\left(\hat{R}^{-1}\right)_{j i}^{l k}$ that this is an involution which is consistent with (3.1), and one can verify that

$$
\Theta^{*}=-\Theta
$$

This also implies

$$
[\omega, \Theta]=0,
$$

hence

$$
\left(d x_{i}\right)^{*}=-\omega d x_{i} \omega^{-1} .
$$

Finally, $*_{H}$ is also compatible with the star structure:

$$
\left(*_{H}(\alpha)\right)^{*}=*_{H}\left(\alpha^{*}\right)
$$

where $\alpha \in \Omega_{q, N}^{1}$, for both $q \in \mathbb{R}$ and $|q|=1$. This is easy to see for $\alpha=\xi_{i}$ in the latter case, and for $\alpha=d x_{i}$ in the case $q \in \mathbb{R}$. This implies that indeed $\left(d \alpha^{(n)}\right)^{*}=d\left(\alpha^{(n)}\right)^{*}$ for all $n$.

We summarize the above results:

Theorem 3.1 The definitions (3.20), (3.22) define a covariant differential calculus on $\Omega_{q, N}^{*}=$ $\oplus_{n=0}^{3} \Omega_{q, N}^{n}$ over $\mathcal{S}_{q, N}^{2}$ with $\operatorname{dim}\left(\Omega_{q, N}^{n}\right)=(1,3,3,1)$ for $n=(0,1,2,3)$. Moreover, this is a *calculus with the star structures (3.27) and (3.31) for $q \in \mathbb{R}$ and $|q|=1$, respectively.

\subsection{Frame formalism}

On many noncommutative spaces [5, 26], it is possible to find a particularly convenient set of one-forms (a "frame") $\theta_{a} \in \Omega^{1}$, which commute with all elements in the function space $\Omega^{0}$. Such a frame exists here as well, and in terms of the $\xi_{i}$ variables, it takes a similar form to that of [5]. Consider the elements

$$
\begin{aligned}
\theta^{a} & =\Lambda_{N} S\left(L_{j}^{+a}\right) g^{j k} \xi_{k} \in \Omega_{q, N}^{1}, \\
\lambda_{a} & =\frac{1}{\Lambda_{N}} x_{i} L_{a}^{+i} \quad \in \mathcal{S}_{q, N}^{2} .
\end{aligned}
$$

where as usual

$$
\begin{aligned}
L_{j}^{+i} & =\mathcal{R}_{1} \pi_{j}^{i}\left(\mathcal{R}_{2}\right), \\
S\left(L^{+i}{ }_{j}\right) & =\mathcal{R}_{1}^{-1} \pi_{j}^{i}\left(\mathcal{R}_{2}^{-1}\right)
\end{aligned}
$$


are elements of $U_{q}(s u(2))$, which we consider here as elements in $\mathcal{S}_{q, N}^{2}$ via (2.31). Then the following holds:

\section{Lemma 3.2}

$$
\begin{aligned}
{\left[\theta^{a}, f\right] } & =0, \\
d f & =\left[\lambda_{a}, f\right] \theta^{a}, \\
\Theta=x_{i} \xi_{j} g^{i j} & =\lambda_{a} \theta^{a} .
\end{aligned}
$$

for any $f \in \mathcal{S}_{q, N}^{2}$. In this sense, the $\lambda_{a}$ are dual to the frame $\theta^{b}$. They satisfy the relations

$$
\begin{aligned}
\lambda_{a} \lambda_{b} g^{b a} & =\frac{1}{q^{4} \Lambda_{N}^{2}} r^{2} \\
\lambda_{a} \lambda_{b} \varepsilon_{c}^{b a} & =-\frac{1}{q^{2}} \lambda_{c} \\
\theta^{a} \theta^{b} & =-q^{2} \hat{R}_{c d}^{b a} \theta^{d} \theta^{c} \\
d \theta^{a} & =\lambda_{b}\left[\theta^{a}, \theta^{b}\right]_{+}+\frac{1}{q^{2}[2]_{q^{2}}} \varepsilon_{b c}^{a} \theta^{c} \theta^{b} \\
*_{H} \theta^{a} & =-\frac{1}{q^{2}[2]_{q^{2}}} \varepsilon_{b c}^{a} \theta^{c} \theta^{b} \\
\theta^{a} \theta^{b} \theta^{c} & =-\Lambda_{N}^{2} \frac{q^{6}}{r^{2}} \varepsilon^{c b a} \Theta^{3}
\end{aligned}
$$

In particular in the limit $q=1$, this becomes $\lambda_{a}=\frac{1}{\Lambda_{N}} x_{a}$, and $d x_{a}=-\varepsilon_{a b}^{c} x_{c} \theta^{b}$, using (3.7). Proof Using

$$
S\left(L^{+i}{ }_{j}\right) x_{k}=x_{l}\left(\hat{R}^{-1}\right)_{j k}^{l n} S\left(L_{n}^{+i}\right)
$$

(which follows from (2.29) ) and $\Delta\left(S\left(L_{j}^{+i}\right)\right)=S\left(L_{j}^{+n}\right) \otimes S\left(L_{n}^{+i}\right)$, it is easy to check that $\left[\theta^{a}, x_{i}\right]=0$ for all $i, a$, and (3.39) follows. (3.41) follows immediately from $L^{+i}{ }_{a} S\left(L_{j}^{+a}\right)=$ $\delta_{j}^{i}$, and To see (3.43), one needs the well-known relation $L_{r}^{+l} L^{+k}{ }_{s} g^{s r}=g^{k l}$, as well as $L_{r}^{+l} L^{+k} \varepsilon_{n}^{s r}=\varepsilon_{m}^{k l} L_{n}^{+m}$; the latter follows from the quasitriangularity of $U_{q}(s u(2))$. The commutation relations among the $\theta$ are obtained as in [5] by observing

$$
\begin{aligned}
\theta^{a} \theta^{b} & =\Lambda_{N} \theta^{a} S\left(L_{n}^{+b}\right) g^{n l} \xi_{l} \\
& =\Lambda_{N} S\left(L^{+b}{ }_{n}\right) \theta^{a} g^{n l} \xi_{l} \\
& =\Lambda_{N}^{2} S\left(L^{+b}{ }_{n}\right) S\left(L^{+a}{ }_{j}\right) g^{j k} g^{n l} \xi_{k} \xi_{l}
\end{aligned}
$$

using the commutation relations $\hat{R}_{i j}^{k l} S L^{+{ }_{n}} S L^{+j}{ }_{m}=S L_{i}^{+k} S L^{+l}{ }_{j} \hat{R}_{n m}^{i j}$, as well as (3.4). The remaining relations can be checked similarly. 


\subsection{Integration of forms}

As classically, it is natural to define the integral over the forms of the highest degree, which is 3 here. Since any $\alpha^{(3)} \in \Omega_{q, N}^{3}$ can be written in the from $\alpha^{(3)}=f \Theta^{3}$, we define

$$
\int \alpha^{(3)}=\int f \Theta^{3}:=\int_{\mathcal{S}_{q, N}^{2}} f
$$

by (2.42), so that $\Theta^{3}$ is the volume form. This definition is natural, since $\left[\Theta^{3}, f\right]=0$. Integrals of forms with degree $\neq 3$ will be set to zero.

This integral satisfies an important cyclic property, as did the quantum trace (2.43). To formulate it, we extend the map $S^{2}$ from $\mathcal{S}_{q, N}^{2}$ to $\Omega_{q, N}^{*}$ by

$$
S^{2}\left(\xi_{i}\right)=q^{-H} \triangleright \xi_{i}
$$

extended as an algebra map. Then the following holds (see Appendix B):

$$
\int \alpha \beta=\int S^{-2}(\beta) \alpha
$$

for any $\alpha, \beta \in \Omega_{q, N}^{*}$ with $\operatorname{deg}(\alpha)+\operatorname{deg}(\beta)=3$. Now Stokes theorem follows immediately:

$$
\int d \alpha^{(2)}=\int\left[\Theta, \alpha^{(2)}\right]=0
$$

for any $\alpha^{(2)} \in \Omega_{q, N}^{2}$, because $S^{2} \Theta=\Theta$. This purely algebraic derivation is also valid on some other spaces [35].

Finally we establish the compatibility of the integral with the star structure. From $\Theta^{*}=-\Theta$ and $(2.45)$, we obtain

$$
\left(\int \alpha^{(3)}\right)^{*}=-\int\left(\alpha^{(3)}\right)^{*} \quad \text { for } q \in \mathbb{R} .
$$

For $|q|=1$, we have to extend the algebra map $\bar{f}(2.48)$ to $\Omega_{q, N}^{*}$. It turns out that the correct definition is

$$
\overline{\xi_{i}}=-q^{-4} g^{i j} \xi_{j}+q^{-2}\left(q-q^{-1}\right) \frac{[2]_{q} C_{N}}{r^{2}} g^{i j} d x_{j}
$$

extended as an antilinear algebra map; compare (3.29) for $q \in \mathbb{R}$. To verify that this is compatible with (3.1) and (3.12) requires the same calculations as to verify the star structure (3.29) for $q \in \mathbb{R}$. Moreover one can check using (3.10) that

$$
\overline{d x_{i}}=-g^{i j} d x_{j}
$$


which implies that $\bar{\Theta}=\Theta$, and

$$
\begin{aligned}
\overline{*_{H}(\alpha)} & =*_{H}(\bar{\alpha}), \\
\overline{d \alpha} & =d \bar{\alpha} \\
\overline{\bar{\alpha}} & =S^{-2} \alpha
\end{aligned}
$$

for any $\alpha \in \Omega_{q, N}^{*}$. Hence we have

$$
\left(\int \alpha^{(3)}\right)^{*}=\int \overline{\alpha^{(3)}} \quad \text { for }|q|=1 .
$$

\section{Actions and fields}

\subsection{Scalar fields}

With the tools provided in the previous sections, it is possible to construct actions for 2dimensional euclidean field theories on the $q$-deformed fuzzy sphere.

We start with scalar fields, which are simply elements $\psi \in \mathcal{S}_{q, N}^{2}$. The obvious choice for the kinetic term is

$$
\begin{aligned}
& S_{k i n}[\psi]=i \frac{r^{2}}{\Lambda_{N}^{2}} \int(d \psi)^{*} *_{H} d \psi \quad \text { for } q \in \mathbb{R}, \\
& S_{k i n}[\psi]=\frac{r^{2}}{\Lambda_{N}^{2}} \int \overline{d \psi} *_{H} d \psi \quad \text { for }|q|=1,
\end{aligned}
$$

which, using Stokes theorem, can equivalently be written in the form

$$
\begin{array}{ll}
S_{k i n}[\psi]=-i \frac{r^{2}}{\Lambda_{N}^{2}} \int \psi^{*}\left(d *_{H} d\right) \psi=-\frac{r^{2}}{\Lambda_{N}^{2}} i \int_{\mathcal{S}_{q, N}^{2}} \psi^{*}\left(*_{H} d *_{H} d\right) \psi & \text { for } q \in \mathbb{R}, \\
S_{k i n}[\psi]=-\frac{r^{2}}{\Lambda_{N}^{2}} \int \bar{\psi}\left(d *_{H} d\right) \psi=-\frac{r^{2}}{\Lambda_{N}^{2}} \int_{\mathcal{S}_{q, N}^{2}} \bar{\psi}\left(*_{H} d *_{H} d\right) \psi & \text { for }|q|=1 .
\end{array}
$$

They are real

$$
S_{k i n}[\psi]^{*}=S_{k i n}[\psi]
$$

for both $q \in \mathbb{R}$ and $|q|=1$, using the reality properties established in the previous sections. 
The fields can be expanded in terms of the irreducible representations

$$
\psi(x)=\sum_{K, n} a^{K, n} \psi_{K, n}(x)
$$

according to (2.28), with coefficients $a^{K, n} \in \mathbb{C}$; this corresponds to the first-quantized case. However, in order to ensure invariance of the actions under $U_{q}(s u(2))$ (or a suitable subset thereof), we must assume that $U_{q}(s u(2))$ acts on products of fields via the $q$-deformed coproduct. This can be implemented consistently only after a "second quantization", such that the coefficients in (4.4) generate a $U_{q}(s u(2))$-module algebra. This will be presented in a forthcoming paper 18$]$.

One can also consider real fields, which have the form

$$
\begin{aligned}
\psi(x)^{*} & =\psi(x) \quad \text { for } q \in \mathbb{R}, \\
\overline{\psi(x)} & =q^{H / 2} \psi(x) q^{-H / 2} \quad \text { for }|q|=1
\end{aligned}
$$

This is preserved under the action of a certain real sector $\mathcal{G} \subset U_{q}(s u(2))$ (4.30); the discussion is completely parallel to the one below (4.32) in the next section, hence we will not give it here.

Clearly $*_{H} d *_{H} d$ is the analog of the Laplace operator for functions, which can also be written in the usual form $d \delta+\delta d$, with $\delta=*_{H} d *_{H}$. It is hermitian by construction. We wish to evaluate it on the irreducible representations $\psi_{K} \in(2 K+1)$, that is, on spin- $K$ representations. The result is the following:

Lemma 4.1 If $\psi_{K} \in \mathcal{S}_{q, N}^{2}$ is a spin $K$ representation, then

$$
*_{H} d *_{H} d \psi_{K}=\frac{2}{[2]_{q} C_{N}}[K]_{q}[K+1]_{q} \psi_{K} .
$$

The proof is in Appendix B.

It is useful to write down explicitly the hermitian forms associated to the above kinetic action. Consider

$$
\begin{aligned}
& S_{k i n}\left[\psi, \psi^{\prime}\right]=i \frac{r^{2}}{\Lambda_{N}^{2}} \int(d \psi)^{*} *_{H} d \psi^{\prime} \quad \text { for } q \in \mathbb{R}, \\
& S_{k i n}\left[\psi, \psi^{\prime}\right]=\frac{r^{2}}{\Lambda_{N}^{2}} \int \overline{d \psi} *_{H} d \psi^{\prime} \quad \text { for }|q|=1 .
\end{aligned}
$$


Using Lemma 2.2, it follows immediately that they satisfy

$$
\begin{aligned}
S_{k i n}\left[\psi, \psi^{\prime}\right]^{*} & =S_{k i n}\left[\psi^{\prime}, \psi\right], \\
S_{k i n}\left[\psi, u \triangleright \psi^{\prime}\right] & =S_{k i n}\left[u^{*} \triangleright \psi, \psi^{\prime}\right]
\end{aligned}
$$

for both $q \in \mathbb{R}$ and $|q|=1$. To be explicit, let $\psi_{K, n}$ be an orthonormal basis of $(2 \mathrm{~K}+1)$. We can be assume that it is a weight basis, so that $n$ labels the weights from $-K$ to $K$. Then it follows that

$$
S_{k i n}\left[\psi_{K, n}, \psi_{K^{\prime}, m}\right]=c_{K} \delta_{K, K^{\prime}} \delta_{n, m}
$$

for some $c_{K} \in \mathbb{R}$. Clearly one can also consider interaction terms, which could be of the form

$$
S_{i n t}[\psi]=\int_{\mathcal{S}_{q, N}^{2}} \psi \psi \psi,
$$

or similarly with higher degree.

\subsection{Gauge fields}

Gauge theories arise in a very natural way on $\mathcal{S}_{q, N}^{2}$. For simplicity, we consider only the analog of the abelian gauge fields here. They are simply one-forms

$$
B=\sum B_{a} \theta^{a} r \quad \in \Omega_{q, N}^{1}
$$

which we expand in terms of the frames $\theta^{a}$ introduced in Section 3.4. Notice that they have 3 independent components, which reflects the fact that calculus is 3 -dimensional. Loosely speaking, the fuzzy sphere does see a shadow of the 3-dimensional embedding space. One of the components is essentially radial and should be considered as a scalar field, however it is naturally tied up with the other 2 components of $B$. We will impose the reality condition

$$
\begin{aligned}
B^{*} & =B \quad \text { for } q \in \mathbb{R}, \\
\bar{B} & =q^{H / 2} B q^{-H / 2} \quad \text { for }|q|=1 .
\end{aligned}
$$

Since only 3-forms can be integrated, the most simple candidates for Langrangians that can be written down have the form

$$
S_{3}=\frac{1}{r^{2} \Lambda_{N}^{2}} \int B^{3}, \quad S_{2}=\frac{1}{r^{2} \Lambda_{N}^{2}} \int B *_{H} B, \quad S_{4}=\frac{1}{r^{2} \Lambda_{N}^{2}} \int B^{2} *_{H} B^{2} .
$$


They are clearly real, with the reality condition (4.12); the factor $i$ for real $q$ is omitted here. We also define

$$
F:=B^{2}-*_{H} B
$$

for reasons which will become clear below. The meaning of the field $B$ becomes obvious if one writes it in the form

$$
B=\Theta+A, \quad B_{a}=\frac{1}{r} \lambda_{a}+A_{a}
$$

While $B$ and $\Theta$ become singular in the limit $N \rightarrow \infty, A$ remains well-defined. Using

$$
\begin{aligned}
F & =d A+A^{2} \\
\int A \Theta^{2} & =\int d A \Theta=\int *_{H} A \Theta, \\
\int A^{2} \Theta & =\frac{1}{2} \int\left(A d A+A *_{H} A\right)
\end{aligned}
$$

which follow from 3.20$)$, one finds

$$
\begin{aligned}
& S_{2}=\frac{1}{r^{2} \Lambda_{N}^{2}} \int A *_{H} A+2 A \Theta^{2} \\
& S_{3}=\frac{1}{r^{2} \Lambda_{N}^{2}} \int A^{3}+\frac{3}{2}\left(A d A+A *_{H} A\right)+3 A \Theta^{2}+\Theta^{3}
\end{aligned}
$$

and

$$
S_{Y M}:=\frac{1}{r^{2} \Lambda_{N}^{2}} \int F *_{H} F=\frac{1}{r^{2} \Lambda_{N}^{2}} \int\left(d A+A^{2}\right) *_{H}\left(d A+A^{2}\right) .
$$

The latter action (which is a linear combination of $S_{2}, S_{3}$, and $S_{4}$ ) is clearly the analog of the Yang-Mills action, which in the classical limit contains a gauge field and a scalar, as we will see below. In the limit $q \rightarrow 1$, it reduces to the action considered in [17.

The actions $S_{3}$ and $S_{2}$ alone contain terms which are linear in $A$, which would indicate that the definition of $A(4.15)$ is not appropriate. However, the linear terms cancel in the following linear combination

$$
S_{C S}:=\frac{1}{3} S_{3}-\frac{1}{2} S_{2}=-\frac{2 \pi}{3 \Lambda_{N}^{2}}+\frac{1}{2} \frac{1}{r^{2} \Lambda_{N}^{2}} \int A d A+\frac{2}{3} A^{3} .
$$

Notice that the "mass term" $A *_{H} A$ has also disappeared. This form is clearly the analog of the Chern-Simons action. It is very remarkable that it exists on $\mathcal{S}_{q, N}^{2}$, which is related to the fact that the calculus is 3 -dimensional. In the case $q=1$, this is precisely what has been found recently in the context of 2-branes on the $S U(2)$ WZW model [2]. 
In terms of the components (4.11), $B^{2}=B_{a} B_{b} \theta^{a} \theta^{b} r^{2}$, and $*_{H} B=-\frac{r}{q^{2}[2]_{q^{2}}} B_{a} \varepsilon_{b c}^{a} \theta^{c} \theta^{b}$. Moreover, it is easy to check that

$$
\begin{aligned}
*_{H}\left(\theta^{b} \theta^{c}\right) & =-q^{2} \varepsilon_{a}^{c b} \theta^{a}, \\
\theta^{a} *_{H} \theta^{b} & =\Lambda_{N}^{2} \frac{q^{4}}{r^{2}} g^{b a} \Theta^{3}, \\
\theta^{a} \theta^{b} *_{H} \theta^{c} \theta^{d} & =[2]_{q^{2}} \Lambda_{N}^{2} \frac{q^{8}}{r^{2}}\left(P^{-}\right)_{a^{\prime} b^{\prime}}^{d c} g^{b^{\prime} b} g^{a^{\prime} a} \Theta^{3}=\Lambda_{N}^{2} \frac{q^{8}}{r^{2}} \varepsilon_{n}^{d c} \varepsilon_{m}^{b a} g^{n m} \Theta^{3} .
\end{aligned}
$$

Hence

$$
\begin{aligned}
F & =\left(B_{a} B_{b}+\frac{1}{q^{2} r[2]_{q^{2}}} B_{c} \varepsilon_{b a}^{c}\right) \theta^{a} \theta^{b} r^{2}=\left(\frac{\lambda_{a}}{r} A_{b}+A_{a} \frac{\lambda_{b}}{r}+A_{a} A_{b}+\frac{1}{q^{2} r[2]_{q^{2}}} A_{c} \varepsilon_{b a}^{c}\right) \theta^{a} \theta^{b} r^{2} \\
& =F_{a b} \theta^{a} \theta^{b} r^{2}
\end{aligned}
$$

where we define $F_{a b}$ to be totally antisymmetric, i.e. $F_{a b}=\left(P^{-}\right)_{b a}^{b^{\prime} a^{\prime}} F_{a^{\prime} b^{\prime}}$ using (3.42). This yields

$$
S_{Y M}=q^{8}[2]_{q^{2}} \int_{\mathcal{S}_{q, N}^{2}} F_{a b} F_{c d}\left(P^{-}\right)_{a^{\prime} b^{\prime}}^{d c} g^{b^{\prime} b} g^{a^{\prime} a}
$$

To understand these actions better, we write the gauge fields in terms of "radial" and "tangential" components,

$$
A_{a}=\frac{x_{a}}{r} \phi+A_{a}^{t}
$$

where $\phi$ is defined such that

$$
x_{a} A_{b}^{t} g^{a b}=0 ;
$$

this is always possible. However to get a better insight, we consider the case $q=1$, and take the classical limit $N \rightarrow \infty$ in the following sense: for a given (smooth) field configuration in $\mathcal{S}_{N}^{2}$, we use the sequence of embeddings of $\mathcal{S}_{q, N}^{2}$ to approximate it for $N \rightarrow \infty$. Then terms of the form $\left[A_{a}^{t}, A_{b}^{t}\right]$ vanish in the limit (since the fields are smooth in the limit). The curvature then splits into a tangential and radial part, $F_{a b}=F_{a b}^{t}+F_{a b}^{\phi}$, where

$$
\begin{aligned}
& F_{a b}^{t}=\frac{1}{2 r}\left(\left[\lambda_{a}, A_{b}^{t}\right]-\left[\lambda_{b}, A_{a}^{t}\right]+A_{c}^{t} \varepsilon_{b a}^{c}\right), \\
& F_{a b}^{\phi}=\frac{1}{2 r^{2}}\left(\varepsilon_{a b}^{c} x_{c} \phi+\left[\lambda_{a}, \phi\right] x_{b}-\left[\lambda_{b}, \phi\right] x_{a}\right) .
\end{aligned}
$$

\footnotetext{
${ }^{7}$ the pull-back of $F$ to the 2 -sphere in the classical case is unaffected by this split
} 
Moreover,

$$
\begin{aligned}
x^{a} F_{a b}^{t} & \rightarrow \frac{1}{4 r}\left[x^{a} \lambda_{a}, A_{b}^{t}\right]-\frac{1}{2 r}\left[\lambda_{b}, x^{a} A_{a}^{t}\right]=0, \\
x^{a}\left[\lambda_{a}, \phi\right] & \rightarrow \frac{1}{2}\left[x^{a} \lambda_{a}, \phi\right]=0
\end{aligned}
$$

in the classical limit, which implies that

$$
\begin{aligned}
\int_{S^{2}} F_{a b}^{t} F^{\phi^{a b}} & =\int_{S^{2}} \frac{1}{2 r^{2}} \varepsilon_{a b}^{n} x_{n} \phi F^{t^{a b}}, \\
\int_{S^{2}} F_{a b}^{\phi} F^{\phi^{a b}} & =\int_{S^{2}} \frac{1}{2 r^{2}}\left(\phi^{2}+\left[\lambda_{a}, \phi\right]\left[\lambda^{a}, \phi\right]\right)
\end{aligned}
$$

in the limit. Therefore we find

$$
S_{Y M}=-\int_{S^{2}}\left(2 F_{a b}^{t} F_{a b}^{t}+\frac{2}{r^{2}} \varepsilon_{a b}^{n} x_{n} \phi F^{t^{a b}}+\frac{1}{r^{2}}\left(\phi^{2}+\left[\lambda_{a}, \phi\right]\left[\lambda^{a}, \phi\right]\right)\right)
$$

in the limit, as in [17]. Similarly, the Chern-Simons action (4.19) becomes

$$
\begin{aligned}
S_{C S} & \rightarrow-\frac{2 \pi}{3 \Lambda_{N}^{2}}+\frac{1}{2 r^{2} \Lambda_{N}^{2}} \int d A^{t}\left(A^{t}+2 \Lambda_{N} \Theta \phi\right)-\Lambda_{N}^{2} \phi^{2} \Theta^{3} \\
& =-\frac{2 \pi}{3 \Lambda_{N}^{2}}+\frac{1}{2 r} \int_{S^{2}} F_{a b}^{t}\left(A_{c}^{t}+2 \frac{x_{c}}{r} \phi\right) \varepsilon^{a b c}-\frac{1}{2 r^{2}} \int_{S^{2}} \phi^{2}
\end{aligned}
$$

for $N \rightarrow \infty$. In the flat limit $r \rightarrow \infty$, the term $F_{a b}^{t} A_{c}^{t} \varepsilon^{a b c}$ vanishes because of (4.26), leaving the $F-\phi$ coupling term (after multiplying with $r$ ).

Back to finite $N$ and $q \neq 1$. To further justify the above definition of curvature (4.14), we consider the zero curvature condition, $F=0$. In terms of the $B$ fields, this is equivalent to

$$
\varepsilon_{c}^{b a} B_{a} B_{b}+\frac{1}{q^{2} r} B_{c}=0
$$

which is (up to rescaling) the same as equation (2.24) with opposite multiplication?; in particular, the solutions $B_{a} \in \mathcal{S}_{q, N}^{2}$ are precisely all possible representations of $U_{q}^{o p}(s u(2))$ in the space of $N+1$-dimensional matrices. They are of course classified by the number of partitions of $\operatorname{Mat}(N+1)$ into blocks with sizes $n_{1}, \ldots, n_{k}$ such that $\sum n_{i}=N+1$, as in the case $q=1$.

\footnotetext{
${ }^{8}$ this can be implemented e.g. using the antipode of $U_{q}(s u(2))$
} 
Gauge invariance. We have seen that actions which describe gauge theories in the limit $q=1$ arise very naturally on $\mathcal{S}_{q, N}^{2}$ (as on certain other higher-dimensional $q$-deformed spaces [34]). However, it is less obvious in which sense they are actually gauge-invariant for $q \neq 1$. For $q=1$, the appropriate gauge transformation is $B \rightarrow U B U^{-1}$, for a unitary element $U \in \mathcal{S}_{N}^{2}$. This transformation does not work for $q \neq 1$, because of (3.46). Instead, we propose the following: let

$$
\begin{aligned}
& \mathcal{H}=\left\{\gamma \in U_{q}(\operatorname{su}(2)): \varepsilon(\gamma)=0, \gamma^{*}=S \gamma\right\}, \\
& \mathcal{G}=\left\{\gamma \in U_{q}(\operatorname{su}(2)): \varepsilon(\gamma)=1, \gamma^{*}=S \gamma\right\}=e^{\mathcal{H}}
\end{aligned}
$$

for $q \in \mathbb{R}$, and

$$
\begin{aligned}
& \mathcal{H}=\left\{\gamma \in U_{q}(s u(2)): \varepsilon(\gamma)=0, \gamma^{*}=S_{0} \gamma\right\}, \\
& \mathcal{G}=\left\{\gamma \in U_{q}(\operatorname{su}(2)): \varepsilon(\gamma)=1, \gamma^{*}=S_{0} \gamma\right\}=e^{\mathcal{H}}
\end{aligned}
$$

for $|q|=1$, where $S_{0}(u)=q^{H / 2} S(u) q^{-H / 2}$. Clearly $\mathcal{H}$ is a subalgebra (without 1 ) of $U_{q}(s u(2)$ ), and $\mathcal{G}$ is closed under multiplication. Using the algebra map $j$ (2.31), $\mathcal{G}$ can be mapped to some real sector of the space of functions on the fuzzy sphere.

Now consider the following "gauge" transformations:

$$
B \rightarrow j\left(\gamma_{(1)}\right) B j\left(S \gamma_{(2)}\right) \quad \text { for } \gamma \in \mathcal{G}
$$

It can be checked easily that these transformations preserve the reality conditions (4.12) for both real $q$ and $|q|=1$. In terms of components $B=B_{a} \theta^{a} r$, this transformation is simply (suppressing $j$ )

$$
B_{a} \rightarrow \gamma_{(1)} B_{a} S \gamma_{(2)}=\gamma \triangleright B_{a},
$$

which is the rotation of the fields $B_{a} \in \mathcal{S}_{q, N}^{2}$ considered as scalar fields $\mathbb{9}$, i.e. the rotation $\gamma \in U_{q}(s u(2))$ does not affect the index $a$ because of (3.39). In terms of the $A_{a}$ variables, this becomes

$$
A_{a} \theta^{a} \rightarrow \gamma_{(1)} A_{a} S \gamma_{(2)} \theta^{a}+\gamma_{(1)} d\left(S \gamma_{(2)}\right)=\left(\gamma \triangleright A_{a}\right) \theta^{a}+\gamma_{(1)} d\left(S \gamma_{(2)}\right)
$$

using (3.6) and (3.39). Hence these transformations are a mixture of rotations of the components (first term) and "pure gauge transformations" (second term). Moreover, the radial and tangential components get mixed.

To understand these transformations better, consider $q=1$. Then we have two transformations of a given gauge field $B_{a}$, the first by conjugation with an unitary element $U \in \mathcal{S}_{q, N}^{2}$,

\footnotetext{
${ }^{9}$ notice that this is not the rotation of the one-form $B$, because $\gamma_{(1)} \xi_{i} S \gamma_{(2)} \neq \gamma \triangleright \xi_{i}$
} 
and the second by (4.32). We claim that the respective spaces of inequivalent gauge fields are in fact equivalent. Indeed, choose e.g. $a=1$; then there exists a unitary $U \in \mathcal{S}_{q, N}^{2}$ such that $U^{-1} B_{a} U$ is a diagonal matrix with real entries. On the other hand, using a suitable $\gamma \in \mathcal{G}$ and recalling (2.28), one can transform $B_{a}$ into the form $B_{a}=\sum_{i} b_{i}\left(x_{0}\right)^{i}$ with real $b_{i}$, which is again represented by a diagonal matrix in a suitable basis. Hence at least generically, the spaces of inequivalent gauge fields are equivalent.

One can also see more intuitively that (4.34) corresponds to an abelian gauge transformation in the classical limit. Consider again $\gamma(x)=e^{i h(x)}$ with $h(x)^{*}=-S h(x)$, approximating a smooth function in the limit $N \rightarrow \infty$. Using properly rescaled variables $x_{i}$, one can see using (2.30) that if viewed as an element in $U(s u(2)), \gamma$ approaches the identity, that is $\gamma \triangleright A_{a}(x) \rightarrow A_{a}(x)$ in the classical limit. Now write the functions on $\mathcal{S}_{N}^{2}$ in terms of the variables $x_{1}$ and $x_{-1}$, for example. Then (2.30) yields

$$
(\mathbf{1} \otimes S) \Delta\left(x_{i}\right)=x_{i} \otimes 1-1 \otimes x_{i},
$$

for $i= \pm 1$, and one can see that

$$
\gamma_{(1)}\left[\lambda_{i}, S \gamma_{(2)}\right] \approx \partial_{i} h\left(x_{i}\right)
$$

in the (flat) classical limit. Hence (4.34) indeed becomes a gauge transformation in the classical limit.

To summarize, we found that the set of gauge transformations in the noncommutative case is a (real sector of a) quotient of $U_{q}(s u(2))$, and can be identified with the space of (real) functions on $\mathcal{S}_{q, N}^{2}$ using the map $j$. However, the transformation of products of fields is different from the classical case. Classically, the gauge group acts on products "componentwise", which means that the coproduct is trivial. Here, we must assume that $U_{q}(s u(2))$ acts on products of fields via the $q$-deformed coproduct, so that the above actions are invariant under gauge transformations, by (2.44). In particular, the "gauge group" has become a real sector of a Hopf algebra. Of course, this can be properly implemented on the fields only after a "second quantization", as in the case of rotation invariance (see Section 4.1). This will be presented in a forthcoming paper [18]. This picture is also quite consistent with observations of a BRST-like structure in $U_{q}(s o(2,3))$ at roots of unity, see [34].

Finally, we point out that the above actions are invariant under a global $U_{q}(s u(2))$ symmetry, by rotating the frame $\theta^{a}$.

\section{$5 \quad$ Drinfel'd twists and the relation with $D$-branes}

Finally we relate our $q$-deformed fuzzy sphere to the effective algebra of functions on spherical $D$-branes in the $S U(2)$ WZW model at level $k$, as determined by Alekseev, Recknagel and 
Schomerus [1]. Their result is as follows. The $D$-branes (more precisely their boundary conditions) are classified by an integer $N$ which satisfies $0 \leq N \leq k$. The Hilbert space of the associated boundary conformal field theory decomposes into irreducible representations of the affine Lie algebra $\widehat{s u(2)}{ }_{k}$. One can assign abstract elements $\left\{Y_{i}^{I}\right\}_{I, i}$ to the boundary vertex operators (primary fields), where $I$ ranges from 0 to $\min (N, k-N)$, and $-I \leq i \leq I$. The $\left\{Y_{i}^{I}\right\}_{i}$ form irreducible spin $I$ representations of the horizontal algebra $s u(2)$, and are interpreted as the analog of spherical harmonics on the $D$-brane; in particular, there exist only finitely many of them. The algebra induced by the OPE of the corresponding boundary vertex operators is given by [1]

$$
Y_{i}^{I} \star Y_{j}^{J}=\sum_{K, k}\left[\begin{array}{ccc}
I & J & K \\
i & j & k
\end{array}\right]\left\{\begin{array}{ccc}
I & J & K \\
N / 2 & N / 2 & N / 2
\end{array}\right\}_{q} Y_{k}^{K} \quad \text { with } q=e^{\frac{i \pi}{k+2}}
$$

where the sum goes from $K=0$ to $\min (I+J, k-I-J, N, k-N)$. This is a finite, noncommutative, quasiassociative algebra $\mathcal{A}$. Here the first bracket denotes the ClebschGordon coefficients of $S U(2)$, and the curly brackets denote the $q$-deformed $6 J$-symbols of $U_{q}(s u(2))$. The latter arise from the fusion matrices of the underlying conformal field theory, which have been known to be related to quantum groups for a long time [3].

In the present paper, we only consider roots of unity $q$ which satisfy (2.36). This means that $N \leq k / 2$ in the above situation, so that we can only consider a certain subset of the allowed boundary conditions here. There will be some qualitative changes in the remaining cases, which we do not consider in the present paper.

The reason for the non-associativity of the algebra $\mathcal{A}$ is a mixing of $q$-deformed and undeformed group theory objects. However as was already indicated in [1], one can sometimes "twist" this algebra using a Drinfeld-twist into an associative one. In particular this can be done if $q$ satisfies (2.36), in the following way: On the same vector space $\mathcal{A}$, we define a new multiplication by

$$
a \tilde{\star} b:=\left(\mathcal{F}^{-1(1)} \triangleright a\right) \star\left(\mathcal{F}^{-1(2)} \triangleright b\right)=\star\left(\mathcal{F}^{-1} \triangleright(a \otimes b)\right) .
$$

Here $a, b \in \mathcal{A}$, and

$$
\mathcal{F}=\mathcal{F}^{(1)} \otimes \mathcal{F}^{(2)} \in U(g) \otimes U(g)
$$

is the Drinfeld twist [10] in Sweedler-notation. We can ignore some fine points here since we only consider certain representations of $\mathcal{F}$. The twist relates the undeformed ClebschGordan coefficients to the deformed ones as follows:

$$
\left[\begin{array}{lll}
I & J & K \\
i & j & k^{\prime}
\end{array}\right]\left(g^{(K)}\right)^{k^{\prime} k}=\left[\begin{array}{ccc}
I & J & K \\
i^{\prime} & j^{\prime} & k^{\prime}
\end{array}\right]_{q}\left(g_{q}^{(K)}\right)^{k^{\prime} k} \pi_{i}^{i^{\prime}}\left(\mathcal{F}^{(1)}\right) \pi_{j}^{j^{\prime}}\left(\mathcal{F}^{(2)}\right)
$$

\footnotetext{
${ }^{10} N$ is denoted by $2 \alpha$ in $[1]$
} 
Here we have raised indices using $\left(g_{q}^{(K)}\right)^{k^{\prime} k}$, which is the $q$-deformed invariant tensor, and we will assume that $\left(g^{(K)}\right)^{k^{\prime} k}=\delta^{k^{\prime} k}$ (in an orthonormal basis). It should be noted that even though the abstract element $\mathcal{F}$ exists only for generic (more precisely formal) $q$, the representations of $\mathcal{F}$ which are needed above do exist at roots of unity, assuming the restrictions (2.36) on $q$; this is because the Clebsch-Gordan decomposition is then still analytic in $q$. Hence the twisted multiplication rule for the generators $Y_{i}^{I}$ is

$$
Y_{i}^{I} \tilde{\star} Y_{j}^{J}=\sum_{K, k}\left[\begin{array}{lll}
I & J & K \\
i & j & k^{\prime}
\end{array}\right]_{q}\left(g_{q}^{(K)}\right)^{k^{\prime} k}\left\{\begin{array}{ccc}
I & J & K \\
N / 2 & N / 2 & N / 2
\end{array}\right\}_{q} Y_{k}^{K} .
$$

As was already pointed out in [1], this defines an associative algebra. We claim that this is precisely the algebra $\mathcal{S}_{q, N}^{2}$, which in turn is the matrix algebra $\operatorname{Mat}(N+1)$. To see this, we reconsider the algebra $\mathcal{S}_{q, N}^{2}$ from a group-theoretic point of view:

Let now $Y_{i}^{I} \in \mathcal{S}_{q, N}^{2}$ be an irreducible spin $I$ representation of $U_{q}(s u(2))$, according to the decomposition (2.28). In acts on the Fock space $\mathcal{F}_{N}(2.16)$, which in turn is a spin $N / 2$ representation of $U_{q}(s u(2))$, with a basis of the form $\left(A^{+} \ldots A^{+}\right)_{r}|0\rangle$. Hence if we denote with $\pi\left(Y_{i}^{I}\right)_{s}^{r}$ the matrix which represents the operator $Y_{i}^{I}$ on $\mathcal{F}_{N}$, we can conclude that it is proportional to the Clebsch-Gordan coefficient of the decomposition $(2 I+1) \otimes(N+1) \rightarrow$ $(N+1)$; this is the Wigner-Eckart theorem. Hence in a suitable normalization of the basis $Y_{i}^{I}$, we can write

$$
\pi\left(Y_{i}^{I}\right)_{s}^{r}=\left(g_{q}^{(N / 2)}\right)^{r r^{\prime}}\left[\begin{array}{ccc}
N / 2 & I & N / 2 \\
r^{\prime} & i & s
\end{array}\right]_{q}=\left[\begin{array}{ccc}
I & N / 2 & N / 2 \\
i & s & r^{\prime}
\end{array}\right]_{q}\left(g_{q}^{(N / 2)}\right)^{r^{\prime} r} .
$$

Therefore the matrix representing the operator $Y_{i}^{I} Y_{j}^{J}$ is given by

$$
\begin{aligned}
\pi\left(Y_{i}^{I}\right)_{s}^{r} \pi\left(Y_{j}^{J}\right)_{t}^{s}=\left(g_{q}^{(N / 2)}\right)^{r r^{\prime}}\left[\begin{array}{ccc}
N / 2 & I & N / 2 \\
r^{\prime} & i & s
\end{array}\right]_{q}\left(g_{q}^{(N / 2)}\right)^{s s^{\prime}}\left[\begin{array}{ccc}
N / 2 & J & N / 2 \\
s^{\prime} & j & t
\end{array}\right]_{q} \\
\quad=\sum_{K}\left\{\begin{array}{ccc}
N / 2 & J & N / 2 \\
I & N / 2 & K
\end{array}\right\}_{q}\left[\begin{array}{lll}
I & J & K \\
i & j & k^{\prime}
\end{array}\right]_{q}\left(g_{q}^{(K)}\right)^{k^{\prime} k}\left[\begin{array}{ccc}
K & N / 2 & N / 2 \\
k & t & r^{\prime}
\end{array}\right]_{q}\left(g_{q}^{(N / 2)}\right)^{r^{\prime} r} \\
\quad=\sum_{K}\left\{\begin{array}{ccc}
I & J & K \\
N / 2 & N / 2 & N / 2
\end{array}\right\}_{q}\left[\begin{array}{lll}
I & J & K \\
i & j & k^{\prime}
\end{array}\right]_{q}\left(g_{q}^{(K)}\right)^{k^{\prime} k} \pi\left(Y_{k}^{K}\right)_{t}^{r} .
\end{aligned}
$$

Here we used the identity

$$
\left\{\begin{array}{ccc}
N / 2 & J & N / 2 \\
I & N / 2 & K
\end{array}\right\}_{q}=\left\{\begin{array}{ccc}
I & J & K \\
N / 2 & N / 2 & N / 2
\end{array}\right\}_{q}
$$


which is proved in 22]. This calculation is represented graphically in Figure 1, which shows that it essentially reduces to the definition of the $6 j$-symbols. Therefore the algebra of $\mathcal{S}_{q, N}^{2}$ is precisely (5.5), which is a twist of the algebra (5.1) found in [1]. In a sense, this twisting is similar to deformation quantization; however, $\mathcal{S}_{q, N}^{2}$ is a $U_{q}(s u(2))$-module algebra, while (5.1) is a $U(s u(2))$-module algebra.

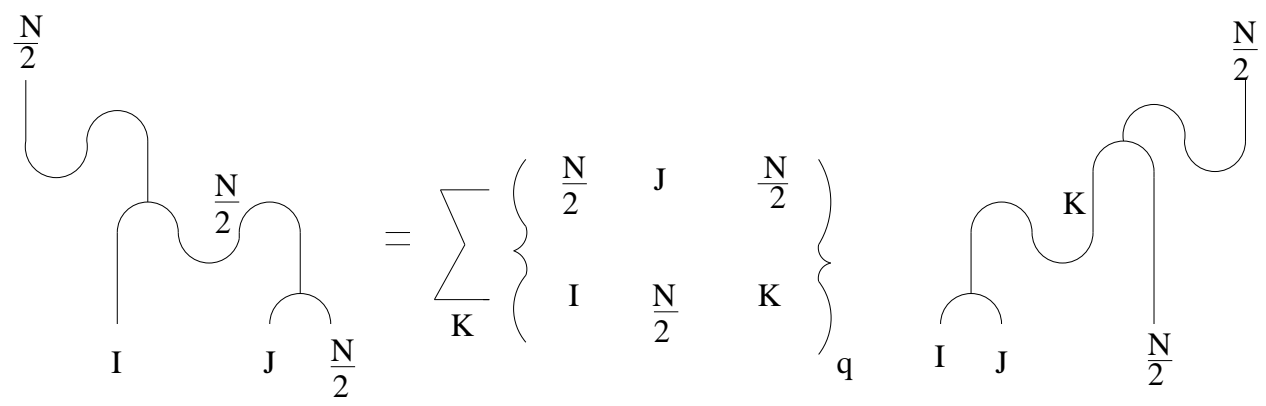

Figure 1: Derivation of the algebra (5.5)

\section{Summary and outlook}

In this paper, we have studied the $q$-deformed fuzzy sphere $\mathcal{S}_{q, N}^{2}$, which is an associative algebra which is covariant under $U_{q}(s u(2))$, for real $q$ and $q$ a phase. In the first case, this is the same as the "discrete series" of Podleś quantum spheres. We develop the formalism of differential forms and frames, as well as integration. We then briefly consider scalar and gauge field theory on this space. It appears that $\mathcal{S}_{q, N}^{2}$ is a nice and perhaps the simplest example of quantum spaces which are covariant under a quantum group. This makes it particularly well suited for studying field theory, an endeavour which has proved to be rather difficult on other $q$-deformed spaces. We are able to write hermitian actions for scalar and gauge fields, including analogs of Yang-Mills and Chern-Simons actions. In particular, the form of the actions for gauge theories suggests a new type of gauge symmetry, where the role of the gauge group is played by $U_{q}(s u(2))$, which can be mapped onto the space of functions on $\mathcal{S}_{q, N}^{2}$. This suggests that formulating field theory on quantized spaces which are less trivial than the ones corresponding to a Moyal product on flat spaces requires new approaches, and may lead to interesting new insights.

The main motivation for doing this is the discovery [1] that a quasi-associative twist of $\mathcal{S}_{q, N}^{2}$ arises on spherical $D$-branes in the $S U(2)_{k}$ WZW model, for $q$ a root of unity. In view 
of this result, we hope that the present formalism may be useful to formulate a low-energy effective field theory induced by open strings ending on the $D$-branes. This in turn inspires one to consider some kind of second quantization of field theories on $\mathcal{S}_{q, N}^{2}$, corresponding to a loop expansion and many-particle states. It is quite interesting that also from a more formal point of view, such a second quantization turns out to be necessary for a satisfactory definition of symmetries in such a field theory. This will be presented in a future publication [18. Moreover, while the question of using either the quasi-associative algebra (5.1) or the associative $\mathcal{S}_{q, N}^{2}$ may ultimately be a matter of taste, the latter does suggest certain forms for Lagrangians, induced by the differential calculus. It would be very interesting to compare this with a low-energy effective action induced from string theory.

In this paper we have only considered spaces which correspond to a subset of the allowed boundary conditions discussed in [1]. The remaining cases will show some qualitatively new features, and are postponed for future work.

Acknowledgements: We would like to thank A. Alekseev, C.-S. Chu, B. Cerchiai, G. Fiore, J. Pawelczyk, E. Scheidegger and J. Wess for useful and stimulating discussions. H. S. and J. M. are grateful for hospitality at the Erwin-Schrödinger Institut in Vienna, where this work was initiated. H. G. and J. M. would also like to thank J. Wess and the Max-Planck Institut für Physik in München for hospitality and financial support.

\section{Appendix A: Invariant tensors}

Before giving the explicit forms of the invariant tensors used in this paper, we briefly explain our conventions and the relation to the literature. The quantum spaces in [14] and in much of the standard literature are defined as left $\operatorname{Fun}_{q}(G)$-comodule algebras. This is equivalent to right $U_{q}(g)$-module algebras. However it is more intuitive to work with left module algebras. This can be achieved using the antipode, $u \triangleright f(x):=f(x) \triangleleft S(u)$; moreover if $\pi_{j}^{i}(u)$ is the fundamental representation, then $u \triangleright x_{i}=x_{j} \pi_{i}^{j}(u)$ where $x_{i}=g_{i j} x^{j}$. However the coproduct then becomes reversed, $u \triangleright f g=\left(u_{2} \triangleright f\right)\left(u_{1} \triangleright g\right)$. We have incorporated this by defining $U_{q}(g)$

with the reversed coproduct (2.9) and antipode. This means that our $\mathcal{R} \in U_{q}^{-} \otimes U_{q}^{+}$(where $U_{q}^{ \pm}$denotes the Borel subalgebras) is obtained from the usual one by flipping the tensor components. For example, our $\hat{R}_{+-}^{+-}=\pi_{+}^{-}\left(\mathcal{R}_{1}\right) \pi_{-}^{+}\left(\mathcal{R}_{2}\right)=\left(q-q^{-1}\right)$ in the fundamental representation of $U_{q}(s u(2))$, where \pm labels the weights. Then the characteristic equation and all compatibility relations with the invariant tensors have the same form as usual, and are obtained from the standard ones by flipping all horizontal indices.

The $q$-deformed epsilon-symbol ("spinor metric") for spin $1 / 2$ representations is given 
by

$$
\varepsilon^{+-}=q^{-\frac{1}{2}}, \quad \varepsilon^{-+}=-q^{\frac{1}{2}},
$$

all other components are zero. The corresponding tensor with lowered indices is $\varepsilon_{\alpha \beta}=-\varepsilon^{\alpha \beta}$ and satisfies $\varepsilon^{\alpha \beta} \varepsilon_{\beta \gamma}=\delta_{\gamma}^{\alpha}$. In particular, $\varepsilon^{\alpha \beta} \varepsilon_{\alpha \beta}=-\left(q+q^{-1}\right)=-[2]_{q}$.

The $q$-deformed sigma-matrices, i.e. the Clebsch-Gordon coefficients for $(3) \subset(2) \otimes(2)$, are given by

$$
\begin{aligned}
& \sigma_{1}^{++}=1=\sigma_{-1}^{--}, \\
& \sigma_{0}^{+-}=\frac{q^{\frac{1}{2}}}{\sqrt{[2]_{q}}}, \quad \sigma_{0}^{-+}=\frac{q^{-\frac{1}{2}}}{\sqrt{[2]_{q}}}
\end{aligned}
$$

in an orthonormal basis, and $\sigma_{i}^{\alpha \beta}=\sigma_{\alpha \beta}^{i}$. They are normalized such that $\sum_{\alpha \beta} \sigma_{\alpha \beta}^{i} \sigma_{j}^{\alpha \beta}=\delta_{j}^{i}$. That is, they define a unitary map (at least for $q \in \mathbb{R}$ ).

The $q$-deformed invariant tensor for spin 1 representations is given by

$$
g^{1-1}=-q^{-1}, \quad g^{00}=1, \quad g^{-11}=-q,
$$

all other components are zero. Then $g_{\alpha \beta}=g^{\alpha \beta}$ satisfies $g^{\alpha \beta} g_{\beta \gamma}=\delta_{\gamma}^{\alpha}$, and $g^{\alpha \beta} g_{\alpha \beta}=q^{2}+1+$ $q^{-2}=[3]_{q}$.

The Clebsch-Gordon coefficients for $(3) \subset(3) \otimes(3)$, i.e. the $q$-deformed structure constants, are given by

$$
\begin{array}{ll}
\varepsilon_{1}^{10}=q^{-1}, & \varepsilon_{1}^{01}=-q, \\
\varepsilon_{0}^{00}=-\left(q-q^{-1}\right), & \varepsilon_{0}^{1-1}=1=-\varepsilon_{0}^{-11}, \\
\varepsilon_{-1}^{0-1}=q^{-1}, & \varepsilon_{-1}^{-10}=-q
\end{array}
$$

in an orthonormal basis, and $\varepsilon_{i j}^{k}=\varepsilon_{k}^{i j}$. They are normalized such that $\sum_{i j} \varepsilon_{i j}^{n} \varepsilon_{m}^{i j}=[2]_{q^{2}} \delta_{m}^{n}$. Moreover, the following identities hold:

$$
\begin{aligned}
\varepsilon_{i j}^{n} g^{j k} & =\varepsilon_{i}^{n k} \\
g_{i j} \varepsilon_{k l}^{j} & =\varepsilon_{i k}^{j} g_{j l} \\
\varepsilon_{i}^{n k} \varepsilon_{k}^{l m}-\varepsilon_{i}^{k m} \varepsilon_{k}^{n l} & =g^{n l} \delta_{i}^{m}-\delta_{i}^{n} g^{l m}
\end{aligned}
$$

which can be checked explicitly. In view of (7.6) , the $q$-deformed totally $(q-)$ antisymmetric tensor is defined as follws:

$$
\varepsilon^{i j k}=g^{i n} \varepsilon_{n}^{j k}=\varepsilon_{n}^{i j} g^{n k} .
$$

It is invariant under the action of $U_{q}(s u(2))$. 


\section{Appendix B: some proofs}

Proof of (3.7): Using the identity

$$
\mathbf{1}=q^{-2} \hat{R}+\left(1+q^{-4}\right) P^{-}+\left(1-q^{-6}\right) P^{0},
$$

(7.5), (3.4), and the braiding relation (3.1) we can calculate the commutation relation of $\Theta$ with the generators $x_{i}$ :

$$
\begin{aligned}
x_{i} \Theta & =x_{i}\left(x_{j} \xi_{t} g^{j t}\right) \\
& =q^{-2} \hat{R}_{i j}^{k l} x_{k} x_{l} \xi_{t} g^{j t}+q^{-2} \Lambda_{N} \varepsilon_{i j}^{n} x_{n} \xi_{t} g^{j t}+\frac{r^{2}}{[3]}\left(1-q^{-6}\right) g_{i j} \xi_{t} g^{j t} \\
& =q^{-2} \Theta x_{i}+q^{-2} \Lambda_{N} \varepsilon_{i j}^{n} x_{n} \xi_{t} g^{j t}+r^{2} \frac{\left(1-q^{-6}\right)}{[3]_{q}} \xi_{i} \\
& =q^{-2} \Theta x_{i}+q^{-2} \Lambda_{N} \varepsilon_{i}^{n k} x_{n} \xi_{k}+r^{2} q^{-3}\left(q-q^{-1}\right) \xi_{i},
\end{aligned}
$$

which yields (3.7).

Proof of (3.13) and (3.15): Using (3.12), one has

$$
\Theta \xi_{i}=-q^{2} \xi_{i} \Theta,
$$

which implies $\Theta \Theta=\Theta(x \cdot \xi)=d x \cdot \xi-q^{2} \Theta \Theta$, hence

$$
\left(1+q^{2}\right) \Theta^{2}=d x \cdot \xi .
$$

On the other hand, (3.7) yields

$$
d x \cdot \xi=-\Lambda_{N} x_{i} \varepsilon_{j}^{k l} \xi_{k} \xi_{l} g^{i j}-q^{3}\left(q-q^{-1}\right) \Theta^{2},
$$

and combining this it follows that

$$
\Theta^{2}=-\frac{q^{2} \Lambda_{N}}{[2]_{q^{2}}} x_{i} \xi_{k} \xi_{l} \varepsilon^{i k l} .
$$

We wish to relate this to $d x_{i} d x_{j} g^{i j}$, which is proportional to $d \Theta$. Using the relations $\varepsilon_{i}^{n k} x_{n} \xi_{k}=$ $-q^{-2} \varepsilon_{i}^{n k} \xi_{n} x_{k}, \Theta=q^{-4} \xi \cdot x$, (7.6) and (7.7), one can show that

$$
\varepsilon_{i}^{n k} x_{n} \xi_{k} \varepsilon_{j}^{m l} x_{m} \xi_{l} g^{i j}=\Lambda_{N} x_{i} \xi_{k} \xi_{l} \varepsilon^{i k l}+q^{2} \Theta^{2}
$$

which using (3.7) implies

$$
d x_{i} d x_{j} g^{i j}=-\frac{1}{C_{N}} r^{2} \Theta^{2} .
$$


Proof of $d \circ d=0$ on $\Omega_{q, N}^{1}$. First, we calculate

$$
\begin{aligned}
{\left[\Theta, d \xi_{i}\right] } & =\left(q^{2}-1\right)\left(q^{2}+1\right)\left(\xi_{i} \Theta^{2}+\frac{q^{-2} \Lambda_{N}}{[2]_{q^{2}}} \varepsilon_{i}^{k l} \xi_{k} \xi_{l} \Theta\right) \\
& =\left(q^{2}-1\right)\left(q^{2}+1\right)\left(\xi_{i}\left(*_{H} \Theta\right)-\left(*_{H} \xi_{i}\right) \Theta\right)=0
\end{aligned}
$$

using (8.2), (3.18), and (3.17). This implies that

$$
\begin{aligned}
d\left(d\left(f \xi_{i}\right)\right) & =\left[\Theta, d f \xi_{i}+f d \xi_{i}\right] \\
& =[\Theta, d f]_{+} \xi_{i}-d f\left[\Theta, \xi_{i}\right]_{+}+d f d \xi_{i}+f\left[\Theta, d \xi_{i}\right] \\
& =d d f+*_{H}(d f) \xi_{i}-d f\left(d \xi_{i}+*_{H}\left(\xi_{i}\right)\right)+d f d \xi_{i} \\
& =-d f *_{H} \xi_{i}+\left(*_{H} d f\right) \xi_{i}=0
\end{aligned}
$$

by (3.17) for any $f \in \mathcal{S}_{q, N}^{2}$. This proves $d \circ d=0$ on $\Omega_{q, N}^{1}$.

Proof of (3.17). First, we show that

$$
\left(*_{H} \xi_{i}\right) \xi_{j}=\xi_{i}\left(*_{H} \xi_{j}\right),
$$

which is equivalent to

$$
\varepsilon_{i}^{n k} \xi_{n} \xi_{k} \xi_{j}=\xi_{i} \varepsilon_{j}^{n k} \xi_{n} \xi_{k} .
$$

Now $\Omega_{q, N}^{3}$ is one-dimensional as module over $\mathcal{S}_{q, N}^{2}$, generated by $\Theta^{3}$ (3.23), which in particular is a singlet under $U_{q}(s u(2))$. This implies that

$$
\begin{aligned}
\varepsilon_{i}^{n k} \xi_{n} \xi_{k} \xi_{j} & =\left(P^{0}\right)_{i j}^{r s} \varepsilon_{r}^{n k} \xi_{n} \xi_{k} \xi_{s} \\
& =-\frac{q^{6}[2]_{q^{2}}}{\Lambda_{N} r^{2}} g_{i j} \Theta^{3} \\
& =\left(P^{0}\right)_{i j}^{r s} \xi_{r} \varepsilon_{s}^{n k} \xi_{n} \xi_{k}=\xi_{i} \varepsilon_{j}^{n k} \xi_{n} \xi_{k},
\end{aligned}
$$

as claimed. Now (3.17) follows immediately using the fact that $*_{H}$ is a left-and right $\mathcal{S}_{q, N^{-}}^{2}$ module map.

Reality structure for $q \in \mathbb{R}$ : These are the most difficult calculations, and they are needed to verify (3.49) as well. First, we have to show that (3.1) is compatible with the star structure (3.29). By a straightforward calculation, one can reduce the problem to proving (3.30). We verify this by projecting this quadratic equation to its spin 0 , spin 1 , and spin 2 part. The first two are easy to check, using (3.10) in the spin 1 case. To show the spin 
2 sector, it is enough to consider (3.30) for $i=j=1$, by covariance. This can be seen e.g. using $\left[x_{1}, \varepsilon_{1}^{i j} x_{i} \xi_{j}\right]=-q^{-2} \Lambda_{N} x_{1} \xi_{1}$, which in turn can be checked using (8.1), (3.3) and (7.4).

Next, we show that (3.12) is compatible with the star structure (3.29). This can be reduced to

$$
\left.\left(q^{2} \hat{R}-q^{-2} \hat{R}^{-1}\right)_{i j}^{k l} d x_{k} \xi_{l}=q^{2}\left(q-q^{-1}\right) \frac{[2]_{q} C_{N}}{r^{2}}\left(\mathbf{1}+q^{2} \hat{R}\right)_{i j}^{k l}\right) d x_{k} d x_{l}
$$

The spin 0 part is again easy to verify, and the spin 1 part vanishes identically (since then $\hat{R}$ has eigenvalue $-q^{-2}$ ). For the spin 2 part, one can again choose $i=j=1$, and verify it e.g. by comparing with the differential of equation (3.30).

Proof of (3.46): Since $\Omega_{q, N}^{*}$ is finitely generated and because of $(2.43)$ and $\left[\Theta^{3}, f\right]=0$, it is enough to consider $\beta=\xi_{k}$. In this case, the claim reduces to

$$
\xi_{i} \xi_{j} \xi_{k}=S^{-2}\left(\xi_{k}\right) \xi_{i} \xi_{j}
$$

Now $S^{-2}\left(\xi_{k}\right)=D_{k}^{l} \xi_{l}$, where $D_{k}^{l}=\delta_{k}^{l} q^{2 r_{l}}$ with $r_{l}=(2,0,-2)$ for $l=(1,0,-1)$, respectively. Since $\xi_{i} \xi_{j}=\frac{1}{[2]_{q^{2}}} \varepsilon_{i j}^{n}\left(\varepsilon_{n}^{r s} \xi_{r} \xi_{s}\right)$, there remains to show that $\left(\varepsilon_{n}^{r s} \xi_{r} \xi_{s}\right) \xi_{k}=S^{-2}\left(\xi_{k}\right)\left(\varepsilon_{n}^{r s} \xi_{r} \xi_{s}\right)$. By (8.4), this is equivalent to

$$
g_{n k} \Theta^{3}=D_{k}^{l} g_{l n} \Theta^{3},
$$

which follows from the definition of $D_{k}^{l}$.

Proof of Lemma 4.1: Using (3.19), (3.24) and $d \Theta^{2}=0$, we have

$$
\begin{aligned}
d *_{H} d \psi & =d\left(\psi \Theta^{2}-\Theta^{2} \psi\right)=(d \psi) \Theta^{2}-\Theta^{2} d \psi \\
& =(d \psi) \Theta^{2}+\left[\Theta^{2}, \psi\right] \Theta \\
& =(d \psi) \Theta^{2}+\left(*_{H} d \psi\right) \Theta .
\end{aligned}
$$

To proceed, we need to evaluate $d \psi_{K}$. Because it is an irreducible representation, it is enough to consider $\psi_{K}=\left(x_{1}\right)^{K}$. From (3.30) and using $\xi_{1} x_{1}=q^{-2} x_{1} \xi_{1}$, it follows that

$$
d x_{1} x_{1}=q^{2} x_{1} d x_{1}-\frac{q^{-2}}{C_{N}} r^{2} x_{1} \xi_{1}
$$

since $\hat{R}$ can be replaced by $q^{2}$ here. By induction, one finds

$$
d x_{1} x_{1}^{k}=x_{1}^{k}\left(q^{2 k} d x_{1}-[k]_{q^{2}} \frac{q^{-2}}{C_{N}} r^{2} \xi_{1}\right),
$$


and by an elementary calculation it follows that

$$
d\left(x_{1}^{k+1}\right)=[k+1]_{q} x_{1}^{k}\left(q^{k} d x_{1}-\frac{q^{-2}}{[2]_{q} C_{N}}[k]_{q} r^{2} \xi_{1}\right) .
$$

Moreover, we note that using (3.17)

$$
\left(\xi_{i} \Theta+*_{H} \xi_{i}\right) \Theta=\xi_{i}\left(*_{H} \Theta\right)+\left(*_{H} \xi_{i}\right) \Theta=2\left(*_{H} \xi_{i}\right) \Theta=-\frac{2}{r^{2}} x_{i} \Theta^{3} .
$$

The last equality follows easily from (8.4) and (8.2). Similarly

$$
\left(d x_{i} \Theta+*_{H} d x_{i}\right) \Theta=2 *_{H} d x_{i} \Theta=2 d x_{i} \Theta^{2} .
$$

Now we can continue (8.5) as

$$
\begin{aligned}
d *_{H} d x_{1}^{K} & =\left(d x_{1}^{K-1} \Theta+*_{H} d x_{1}^{K-1}\right) \Theta \\
& =[K]_{q} x_{1}^{K-1}\left(2 q^{K-1} d x_{1} \Theta^{2}-2 \frac{q^{-2}}{[2]_{q} C_{N}}[K-1]_{q} x_{1} \Theta^{3}\right) .
\end{aligned}
$$

Finally it is easy to check that

$$
d x_{i} \Theta^{2}=-\frac{1}{C_{N}} x_{i} \Theta^{3},
$$

and after a short calculation one finds (4.6).

\section{References}

[1] A. Yu. Alekseev, A. Recknagel, V. Schomerus, "Non-commutative World-volume Geometries: Branes on SU(2) and Fuzzy Spheres", JHEP 9909, 023 (1999).

[2] A. Yu. Alekseev, A. Recknagel, V. Schomerus, "Brane Dynamics in Background Fluxes and Non-commutative Geometry", hep-th/0003187.

[3] L. Alvarez-Gaumé, C. Gomez, G. Sierra, "Duality and Quantum Groups" Nucl. Phys. B330, 347 (1990)

[4] J. Apel, K. Schmüdgen, "Classification of Three-Dimensional Covariant Differenial Calculi on Podleś' Quantum Spheres and on Related Spaces", Lett. Math. Phys. 32, 25 (1994); 
[5] B. Cerchiai, G. Fiore, J. Madore, "Geometrical Tools for Quantum Euclidean Spaces", math/0002007;

[6] B. Cerchiai, J. Madore, S. Schraml, J. Wess, "Structure of the Three-dimensional Quantum Euclidean Space", math/0004011;

[7] M. Douglas, C. Hull, "D-branes and the Noncommutative Torus", JHEP 9802 (1998) 008 ;

C.-S. Chu, P.-M. Ho, "Noncommutative Open String and D-brane", Nucl.Phys. B550 (1999) 151; hep-th/9812219;

N. Seiberg, E. Witten, "String Theory and Noncommutative Geometry", JHEP 9909 (1999) 032; hep-th/9908142

[8] A. Connes, "Noncommutative Geometry", Academic Press, 1994.

[9] V. Drinfeld, "Quantum Groups" Proceedings of the International Congress of Mathematicians, Berkeley, 1986 A.M. Gleason (ed.), p. 798, AMS, Providence, RI

[10] V. G. Drinfeld, "Quasi-Hopf Algebras", Leningrad Math. J. 1, No.6, 1419 (1991);

V. G. Drinfeld, "On Quasitriangular Quasi-Hopf algebras and a group closely connected with $\operatorname{Gal}(\bar{Q} / Q)$ ", Leningrad Math. J. 2, No.4, 829 (1991);

[11] A. Dimakis and J. Madore, "Differential calculi and linear connections," J. Math. Phys. 37, no. 9, 4647 (1996); q-alg/9601023.

[12] M. Dubois-Violette, R. Kerner, and J. Madore, "Gauge bosons in a noncommutative geometry," Phys. Lett. B217, 485 (1989).

[13] T. Filk, "Divergences in a Field Theory on Quantum Space", Phys. Lett. B376, 53 (1996);

S. Minwalla, M. V. Raamsdonk, N. Seiberg, "Noncommutative Perturbative Dynamics", hep-th/9912072;

T. Krajewski, R. Wulkenhaar, "Perturbative quantum gauge fields on the noncommutative torus", hep-th/9903187

[14] L.D.Faddeev, N.Yu.Reshetikhin, L.A.Takhtajan. "Quantization of Lie Groups and Lie Algebras" Algebra Anal. 1178 (1989)

[15] G. Fiore, "The $S O_{q}(N)$ - Symmetric Harmonic Oscillator on the Quantum Euclidean Space $R_{q}^{N}$ and its Hilbert Space Structure" Int. J. Mod. Phys A8, 4679 (1993) 
[16] H. Grosse, C. Klimcik, P. Presnajder, "Towards Finite Quantum Field Theory in Non-Commutative Geometry", Int. J. Theor. Phys. 35, 231 (1996), hep-th/9505175; H. Grosse, C. Klimcik, P. Presnajder, "Field Theory on a Supersymmetric Lattice", Commun. Math. Phys. 185, 155 (1997), hep-th/9507074.

[17] H. Grosse, J. Madore, "A Noncommutative Version of the Schwinger Model", Phys.Lett. B 283: 218 (1992)

[18] H. Grosse, J. Madore, H. Steinacker, in preparation

[19] M. Jimbo, "A q - Difference Analogue of $U(g)$ and the Yang - Baxter Equation" Lett. Math. Phys. 10, 63 (1985)

[20] G. Keller, "Fusion Rules of $U_{q}(s l(2, \mathbb{C})), q^{m}=1 "$. Lett. Math. Phys. 21, 273 (1991)

[21] A.N. Kirillov, N. Reshetikhin, "q- Weyl group and a Multiplicative Formula for Universal R- Matrices" Comm. Math. Phys. 134, 421 (1990)

[22] A.N. Kirillov, N. Reshetikhin, "Representations of the algebra $U_{q}(s l(2))$, $q$-orthogonal polynomials and invariants of links", LOMI preprint E-9-88; in Kohno, T. (ed.): New developments in the theory of knots, World Scientific, 1989 202-256.

[23] S.Z. Levendorskii, Y.S. Soibelman, "Some applications of the quantum Weyl group" Journ. Geom. Phys 7 (2), 241 (1990)

[24] G. Mack, V. Schomerus, "Quasi Hopf quantum symmetry in quantum theory" Nucl. Phys. B 370, 185 (1992)

[25] J. Madore, "The Fuzzy Sphere", Class. Quant. Grav. 9, 69 (1992)

[26] J. Madore, "An introduction to noncommutative differential geometry and its physical applications". No. 257 in London Mathematical Society Lecture Note Series. Cambridge University Press, second ed., 1999.

[27] J. Madore, H. Steinacker, "Propagator on the h-deformed Lobachevsky plane", J.Phys. A33 (2000) 327-342; math/9907023

[28] O. Ogievezky and B. Zumino, "Reality in the Differential Calculus on $q$ - Euclidean Spaces." Lett. Math. Phys. 25, 121 (1992)

[29] P. Podleś, "Quantum spheres", Lett. Math. Phys. 14, 193 (1987). 
[30] P. Podleś, "Differential Calculus on Quantum Spheres", Lett. Math. Phys. 18, 107 (1989).

[31] N. Reshetikhin, "Quantized universal enveloping algebras, the Yang-Baxter equation and invariants of links I,II" LOMI preprint E-4-87, E-17-87.

[32] M. Rosso, "Finite Dimensional Representations of the Quantum Analog of the Enveloping Algebra of a Complex Simple Lie Algebra" Comm. Math. Phys. 117, 581 (1988)

[33] H. Steinacker, "Quantum Anti-de Sitter space and sphere at roots of unity", Adv. Theor. Math. Phys. 4, Nr. 1 (2000); hep-th/9910037.

[34] H. Steinacker, "Quantum Groups, Roots of Unity and Particles on quantized Anti-de Sitter Space". Ph.D. Thesis, Berkeley, May 1997; hep-th/970521];

H. Steinacker, "Unitary Representations and BRST Structure of the Quantum Antide Sitter Group at Roots of Unity", Proceedings to WigSym5, Vienna, Austria, 25-29 August, 1997; a-alg/9710016.

[35] H. Steinacker, "Integration on quantum Euclidean space and sphere in $N$ dimensions" J. Math. Phys. 37 Nr.9, 7438 (1996); q-alg/9506020

[36] J. Wess, B. Zumino, "Covariant Differential Calculus on the Quantum Hyperplane" Nucl Phys 18B (Proc. Suppl.), 302 (1990); U. Carow-Watamura, M. Schlieker, S. Watamura, Z. Phys. C Part. Fields 49, 439 (1991)

[37] S.L. Woronowicz, "Differential Calculus on Compact Matrix Pseudogroups". Comm. Math. Phys. 122, 125 (1989) 